\title{
Evolutionary Population Dynamics and Grasshopper Optimization Approaches for Feature Selection Problems
}

\author{
Majdi Mafarja ${ }^{\mathrm{a}}$, Ibrahim Aljarah ${ }^{\mathrm{b}}$, Ali Asghar Heidaric ${ }^{\mathrm{c}}$, Abdelaziz I. \\ Hammouri $^{\mathrm{d}}$, Hossam Faris ${ }^{\mathrm{b}}$, Ala' M. Al-Zoubi ${ }^{\mathrm{b}}$, Seyedali Mirjalilie \\ ${ }^{a}$ Department of Computer Science, Birzeit University, Birzeit, Palestine, \\ mmafarja@birzeit.edu,mmafarjeh@gmail.com \\ ${ }^{b}$ King Abdullah II School for Information Technology, The University of Jordan, \\ Amman, Jordan \\ \{hossam.faris,i.aljarah\}@ju.edu.jo, alaah14@gmail.com \\ ${ }^{c}$ School of Surveying and Geospatial Engineering, University of Tehran, Tehran, Iran \\ as_heidari@ut.ac.ir \\ ${ }^{d}$ Department of Computer Information Systems, Al-Balqa Applied University, Al-Salt, \\ Jordan \\ Aziz@bau.edu.jo \\ ${ }^{e}$ School of Information and Communication Technology, Griffith University, Nathan, \\ Brisbane, QLD 4111, Australia \\ seyedali.mirjalili@griffithuni.edu.au
}

\begin{abstract}
Searching for the optimal subset of features is known as a challenging problem in feature selection process. To deal with the difficulties involved in this problem, a robust and reliable optimization algorithm is required. In this paper, grasshopper optimization algorithm (GOA) is employed as a search strategy to designa wrapper-based feature selection method. The GOA is a recent population-based metaheuristic that mimics the swarming behaviors of grasshoppers. In this work, an efficient optimizer based on the simultaneous use of the GOA, selection operators, and Evolutionary Population Dynamics (EPD) is proposed in the form of four different strategies to mitigate the immature convergence and stagnation drawbacks of the conventional GOA. In the first two approaches, one of the top three agents and a randomly generated one are selected to reposition a solution from the worst half of the population. In the third and fourth approaches, to give a chance to the low fitness solutions in reforming the population, Roulette Wheel Selection (RWS) and
\end{abstract}


Tournament Selection (TS) are utilized to select the guiding agent from the first half. The proposed GOA_EPD approaches are employed to tackle various feature selection tasks. The proposed approaches are benchmarked on 22 UCI datasets. The comprehensive results and various comparisons reveal that the EPD has a remarkable impact on the efficacy of the GOA and using the selection mechanism enhanced the capability of the proposed approach to outperform other optimizers and find the best solutions with improved convergence trends. Furthermore, the comparative experiments demonstrate the superiority of the proposed approaches when compared to other similar methods in the literature.

Keywords: Grasshopper Optimization Algorithm, GOA, Feature Selection, Classification, Metaheuristics, Evolutionary Population Dynamics, Binary

\section{Introduction}

The existence of thousands of applications of information systems complicated the role of extracting useful information from the collected data $[1,2]$. Data mining plays the main role in extracting the useful knowledge from the collected datasets $[3,4]$. The collected datasets may contain irrelevant and redundant data. Feature selection (FS) is one of the major preprocessing phases that aims to exclude the irrelevant/redundant data from the dataset being processed $[5,6]$.

FS methods can be broadly categorized into three main classes: supervised [7], unsupervised [8], and semi-supervised methods [9]. Supervised FS requires the availability of the class labels to select proper features and used for classification problems. While in unsupervised FS, the class labels are not required, and used for clustering tasks. On the other hand, semi-supervised methods applied when part of the data is labeled.

There are several supervised, semi supervised, and unsupervised FS algorithms in literature. To name a few, the correlation-based feature selection (CFS) [7], fast correlation-based filter (FCBF) [10], and wavelet power spectrum (Spectrum) [11] are examples on supervised techniques. While non-negative spectral learning and sparse regression-based dual-graph regularized (NSSRD) feature selection is one of the latest unsupervised techniques proposed by Shang et al. in 2017 [8]. The subspace learning-based graph regularized (SGFS) technique and self-representation based dual-graph regularized feature selection clustering (DFSC) are also well-established FS 
techniques proposed by Shang et al. in 2016 [12, 13]. On the other hand, feature selection via spectral analysis, and forward feature selection $[9,14]$ are examples on semi-supervised FS.

FS process can be accomplished in four major steps [15]: subset generation, subset assessment, ending criterion, and validation. From the evaluation perspectives, FS methods can be divided to two groups based on selection strategy: wrapper-based and filter-based. In filter-based methods, the selection of a subset is performed independently from the learning algorithm (e.g., classification). The merits of a feature or a subset of them is estimated with regard to specific characteristics of the info [16]. Examples of filter models include Chi-Square [17], Information Gain (IG) [18], Gain Ratio [19], and ReliefF [20]. In the wrapper-based methods, the goodness of a subset is evaluated based on a learning algorithm [21]. Examples of wrapper models include the LVW algorithm [22] and a neural network-based method [23].

Subset generation is considered as a search process to select a subset of items from the initial set using complete, heuristic search, or a random search $[15,24,25]$. The complete search generates all possible subsets to select the best one. If the dataset includes $n$ features, then $2^{n}$ subsets will be generated and assessed, which is computationally expensive for the larger size datasets. Random search is another possible policy to select the attributes. It searches for the next feature subset randomly [26]. The main drawback of the random search strategy is that it may perform as a complete search in the worst case $[5,27]$.

An alternative strategy to the previous two strategies is the heuristic search. Heuristic search can be clarified as a 'depth first' search managed by heuristics. According to Talbi [27], metaheuristic search methods can be defined as "upper level general methodologies (templates) that can be used as guiding strategies in designing underlying heuristics to solve specific optimization problems"[27]. Various metaheuristics such as Grey wolf optimizer (GWO) [28, 29], Whale Optimization Algorithm (WOA) [30], Ant Lion Optimization (ALO) [31], Firefly Algorithm (FA) [32], Particle Swarm Optimization (PSO) [33], and Ant Colony Optimization (ACO) [34] may demonstrate superior efficiencies in tackling feature selection problems when compared to the exact methods $[35,36]$. Metaheuristic algorithms have shown improved results and efficiencies in dealing with many real-life applications such as path planning [37], clustering [38], and power dispatch [39]. For example, E.S. Ali et al. applied the ALO to find the best location and sizing of renewable distributed generations [40]. Wu et al. utilized the WOA for path 
planning of solar-powered UAV [37]. Faris et al. also reviewed the recent variants and applications of the GWO [41].The history of metaheuristics is presented in [42].

The GOA is a new efficient nature-inspired population-based metaheuristic algorithm [43] proposed by Saremi et al. in 2017 to inspire the idealized swarming behaviors of grasshopper insects in nature. This algorithm can disclose improved results and efficiencies on global unconstrained/constrained optimization and various real-life tasks. The basic GOA has been applied to realize the best parameters of proton exchange membrane fuel cells (PEMFCs) stack and the results exposed the viability of the GOA-based algorithm in dealing with the steady-state and dynamic models [44]. In 2017, Wu et al. [45] proposed a dynamic GOA for optimizing the distributed trajectory of UAVs in urban environments. They proved that this algorithm can attain enhanced results and satisfactory trajectories. Tharwat et al. [46] developed a modified multi-objective GOA (MOGOA) with external archive for constrained and unconstrained problems. Mirjalili et al. [47] also developed the basic multi-objective GOA and revealed that the proposed algorithm can tackle several benchmark problem, effectively and with better performance in terms of accuracy of Pareto optimal solutions and the related distribution.

Although the metaheuristic algorithms do not guarantee finding the best solution in all runs, they can find relatively accurate solutions in a reasonable time [27, 48]. Metaheuristics can be classified into two main families; single-solution and population-based algorithms [27]. In the former class (e.g., Simulated Annealing), one solution is manipulated and transformed during the search process, while a set of solutions is evolved in the former class (e.g., PSO). Single-solution-based algorithms show more exploitative behaviour; which means digging the space around a possible solution whereas the population-based class are more explorative or a mix of both behavior; which means exploring different regions of the space [27]. When designing a metaheuristic algorithm, these two criteria should be taken into account. High exploration decreases the quality of results and causes an unpromising convergence. This results in a failure to find the target global optimum. However, high exploitation may cause the optimizer to be trapped in Local Optima (LO).

Evolutionary algorithms (EA) are deep-rooted metaheuristics inspired by natural processes [49, 50]. Genetic algorithms (GA), by J. H. Holland [51]; and evolutionary programming by L. Fogel et.al [52] are two different kind of EA. In recent years, many EA are proposed to tackle the optimization 
problems especially in the field of feature selection [53, 54, 55]. Ant Colony (AntRSAR) and Genetic Algorithm (GenRSAR) are two EAs that have been proposed by Jensen and Shen $[56,57]$ and applied to FS problems. For instance, a chaos-based genetic FS method (CGFSO) has been proposed in [58]. Two hybrid approaches have been proposed in [59] between the GA and Simulated Annealing (SA) and in [60] between the GA and Record to Record algorithm. A Scatter Search-based approach (SSAR) proposed by Jue et al. [61] is another EA-based FS method. Ant Lion Optimizer (ALO), a recent well-regarded metaheuristic, proposed by S. Mirjalili in [62], was utilized as a searching mechanism in a wrapper FS method in $[63,64]$. A chaotic ALO approach was proposed for FS in [65]. The GWO, as another recent population-based optimizer [29], has been successfully employed to tackle several applications like the tuning of fuzzy control systems [66]. It has been applied to FS problems $[67,68]$ as well. Recently, a new wrapper-based FS algorithm that uses a hybrid Whale Optimization algorithm (WOA) with SA algorithm as a search method was proposed in [69].

EAs are modeled to mimic the evolution of individuals from their initial states to become better adapted to some objectives imposed upon them. These revolutionary paradigms apply some evolutionary operators (mutation and recombination in GA or pheromone updating rules of $\mathrm{ACO}$ ) to some selected individuals (based on some selection mechanisms; random, tournament, and roulette wheel selection) in the population to generate an offspring. However, these operators affect and manipulate individuals rather that the whole population. Evolutionary Population Dynamics (EPD) is another evolutionary operator that manipulates the whole population rather than manipulating individuals [70]. Using this operator with EAs will omit the worst individuals from the population rather than improving the best individuals in the population (e.g., recombination in GA). Extremal optimization (EO) [71] is a metaheuristic algorithm that works based on the idea of EPD. The EO algorithm has been used in many research fields with much success [72, 73, 74]. The EPD operator is the main feature that enhanced the performance of this algorithm [28].

This paper presents an efficient GOA-based optimizer with EPD and selection operators are proposed to improve the efficacy of the basic GOA in dealing with FS tasks. In this work, we have made the following key contributions:

- The significant merits of the EPD operator motivated our attempts to 
apply it to the recently proposed Grasshopper Optimization Algorithm (GOA) and investigate its effectiveness on FS problems.

- Four variants of GOA with EPD operator are proposed. In the first two approaches, one of the top three solutions and a randomly generated solution are selected to reposition a solution from the worst half of the population. In the third and fourth approaches, to give a chance to the low fitness solutions to reformulate the population, two different selection mechanisms (namely Roulette Wheel Selection (RWS) and Tournament Selection (TS) are utilized to select the guiding solution from the first half.

- The proposed approaches have been tested on 22 real benchmarks datasets to show its efficiency for feature selection tasks.

- The hybrid GOA and EPD operator is proposed for the first time to solve the feature selection tasks.

- The proposed GOA based approaches have been tested on real datasets with different settings and characteristics to demonstrate its effectiveness and quality of solutions.

The rest of this paper is organized as follows: Section 2 presents a background about EPD operator. The basics of the GOA algorithm and the hybridization with EPD operator is given in Section 2 as well. Section 3 presents the details of the proposed approaches. In Section 4, the experimental results are presented and results are analysed. Finally, in Section 5, conclusions and future work are given. Table 1 describes all the abbreviations used in this paper.

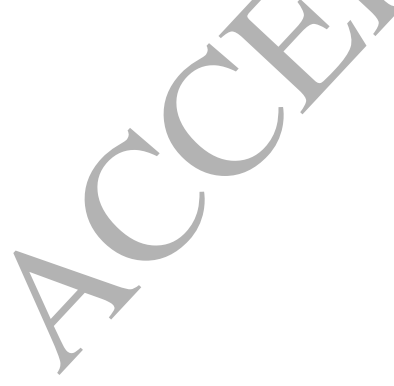


Table 1: List of abbreviations

\begin{tabular}{|c|c|}
\hline Abbreviations & Expansions \\
\hline Acc & Accuracy \\
\hline $\mathrm{ACO}$ & Ant Colony Optimization \\
\hline ALO & Ant Lion Optimizer \\
\hline Atts & Attributes \\
\hline BGOA & Binary GOA \\
\hline BGWO & Binary GWO \\
\hline CFS & Correlation-based Feature Selection \\
\hline CGFSO & Chaos-based Genetic FS Method \\
\hline $\mathrm{CM}$ & Crossover and Mutation \\
\hline DFSC & Self-representation Based Dual-graph Regularized Clustering \\
\hline EA & Evolutionary Algorithms \\
\hline $\mathrm{EO}$ & Extremal Optimization \\
\hline $\mathrm{EPD}$ & Evolutionary Population Dynamics \\
\hline EPSCO & Evolutionary Programming using Self-Organizing $\mathrm{Cr}$ \\
\hline FA & Firefly Algorithm \\
\hline FCBF & Fast Correlation-based Filter \\
\hline FS & Feature Selection \\
\hline F-score & Fisher Score \\
\hline GOA & Grasshopper Optimization Algorithm \\
\hline GWO & Grey Wolf Optimizer \\
\hline GA & Genetic Algorithms \\
\hline IG & Information Gain \\
\hline$k-\mathrm{NN}$ & $k$-Nearest Neighbor \\
\hline LO & Local Optima \\
\hline NSSRD & Non-negative Spectral Learning and Sparse Regression-based Dual-graph Regularized \\
\hline PSO & Particle Swarm Optimization \\
\hline RWS & Roulette Wheel Selection \\
\hline $\mathrm{SA}$ & Simulated Annealing \\
\hline SGFS & Subspace Learning-based Graph Regularized \\
\hline SOC & Self-Organized Criticality \\
\hline SSAR & Scatter Search-based Approach \\
\hline StdDev & Standard Deviation \\
\hline TS & Tournament Selection \\
\hline Ts & Tournament Size \\
\hline WOA & Whale Optimization Algorithm \\
\hline
\end{tabular}

\section{EPD for The GOA}

\subsection{Evolutionary Population Dynamics (EPD)}

EAs are known as stochastic search methods in which a set of solutions (population) is initialized and then gradually improved to become better adapted to the objectives imposed upon them. Some EAs utilize mutation mechanisms to alter the selected solutions, while others employ the crossover operators. These operators aim to evolve the top selected solutions that are mostly the best solutions. The EPD is the process of eliminating the worst solutions in a population by repositioning them around the best ones. The EPD is basically based on the theory of self-organized criticality (SOC) [75] which 
indicates that a local change in the population may affect the whole population and provide delicate balances without external organising force [70]. In the GA, the best solutions are combined using the evolutionary operators (crossover and mutation). In contrast, in the EPD, the worst solutions should be omitted from the current population. Evolutionary programming using self-organizing criticality (EPSCO) [76] and Extremal optimization (EO) [71] are two metaheuristics methods that were proposed based on the SCO concept. The EPD is a simple and effective mechanism that can be embedded in different optimizers. It starts by removing the worst solutions from the swarm and then repositioning the removed solutions around the best search agents.

\subsection{Grasshopper Optimization Algorithm (GOA)}

The GOA is a recent swarm-based nature-inspired algorithm [43] proposed by Saremi et al. It mimics the idealized swarming behavior of grasshopper insects in nature. Similarly other population-based algorithms [77, 78], in GOA, a set of candidate solutions (each individual represents a grasshopper) are randomly generated to construct the initial artificial swarm. Next, all candidate agents are evaluated with regard to the fitness values and the best search agent in the current swarm in considered as the target or leader. The target grasshopper starts attracting the other individuals around its location, and all grasshoppers start moving towards the target grasshopper.

The movement of the $i$-th grasshopper towards the target grasshopper is denoted as $X_{i}$ and is formulated as in Eq. (1).

$$
X_{i}=S_{i}+G_{i}+A_{i}
$$

where $S_{i}$ is the social interaction, $G_{i}$ is the gravity force on $i$-th grasshopper, and $A_{i}$ shows the wind advection. The social interaction $S_{i}$ acts as the main component during the grasshopper movement process. It can be calculated as Eq. (2):

$$
S_{i}=\sum_{j=1, j \neq i}^{N} s\left(d_{i j}\right) \widehat{d_{i j}}
$$

where $d_{i j}$ is the Euclidian distance of the $i$-th with the $j$-th grasshopper, and it is calculated as $d_{i j}=\left|x_{j}-x_{i}\right|$. While, $\widehat{d_{i j}}=\frac{x_{j}-x_{i}}{d_{i j}}$ is a unit vector from the $i$-th grasshopper to the $j$-th grasshopper. The $s$ function is defined as the 
strength of social forces, which can be calculated as follows:

$$
s(r)=f e^{\frac{-d}{l}}-e^{-d}
$$

where $f$ is the intensity of attraction and $l$ is the attractive length scale.

Fig. 1 illustrates the impact of $s$-function on the attraction and repulsion (i.e., social interaction) of the grasshoppers. In this figure, the distance $d$ has been considered in the interval of $[0,15]$. The repulsion force between grasshoppers occurs when the distance between them is between 0 and 2.079 units. In the case that the distance between a grasshopper and other agents is 2.079 , it enters to the comfort zone, where neither attraction nor repulsion occurs there, while the attraction starts increasing after 2.079 till 4 and then starts decreasing.

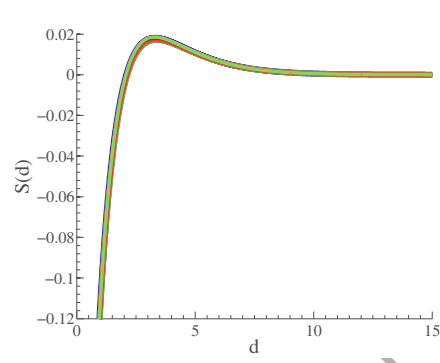

(a) When $l=1.5$ and $f=0.5$

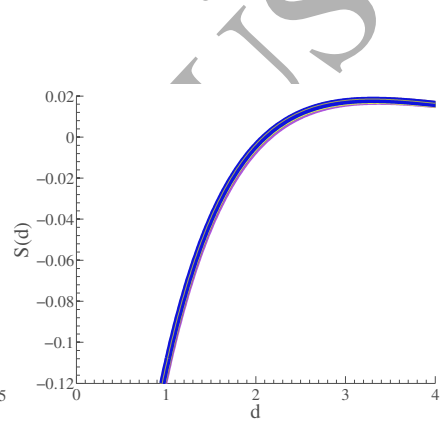

(b) When $d$ is inside $[0,4]$

Figure 1: Function $s$ when $f=1.5$ and $f=0.5$ and closer window when $d$ changes in $[0,4]$.

Fig. 1 shows that while the distance between grasshoppers becomes larger, $s$-function returns values close to 0 . Thus, for large distances between grasshoppers, $s$-function is not capable of applying strong forces to them. To overcome this drawback, the distance between agents are mapped between 1 and 4 . The shape of the $s$-function in the interval $[1,4]$ is shown in Fig. 1 (right).

Different social behaviors can be obtained for the artificial grasshoppers by changing the parameters $l$ and $f$ of $s$-function in Eq. (3) as shown in Fig. 2.

The conceptual model of the comfort zone and the attraction and repulsion forces between the grasshoppers is also shown in Fig. 3.

The gravity force $G_{i}$ (second component in Eq. (4)) is calculated as follows:

$$
G_{i}=-g \times \widehat{e_{g}}
$$




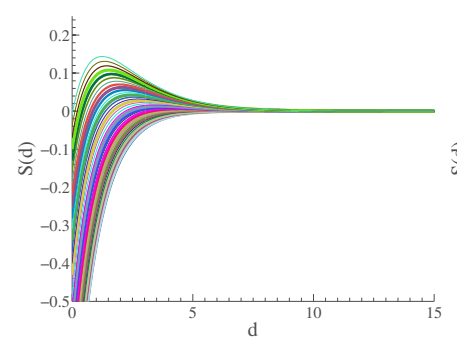

(a) $l=1.5$ and $f$ in $[0,1]$

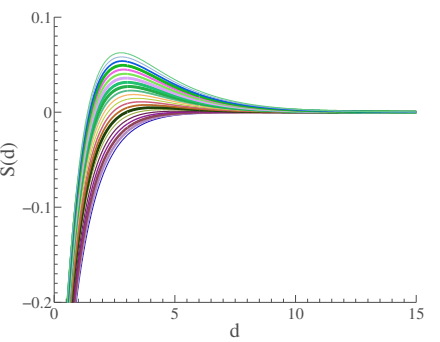

(b) $f=0.5$ and $l$ in $[1,2]$

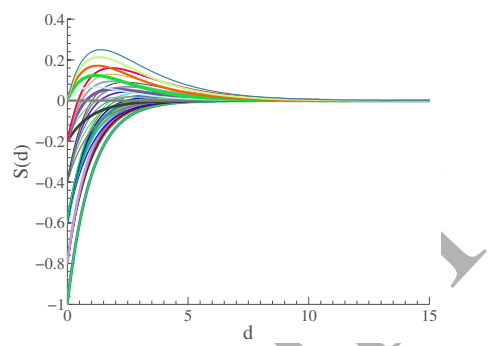

(c) $f$ in $[0,1], l$ in $[1,2]$

Figure 2: behavior of the function $s$ based on $l$ and $f$.

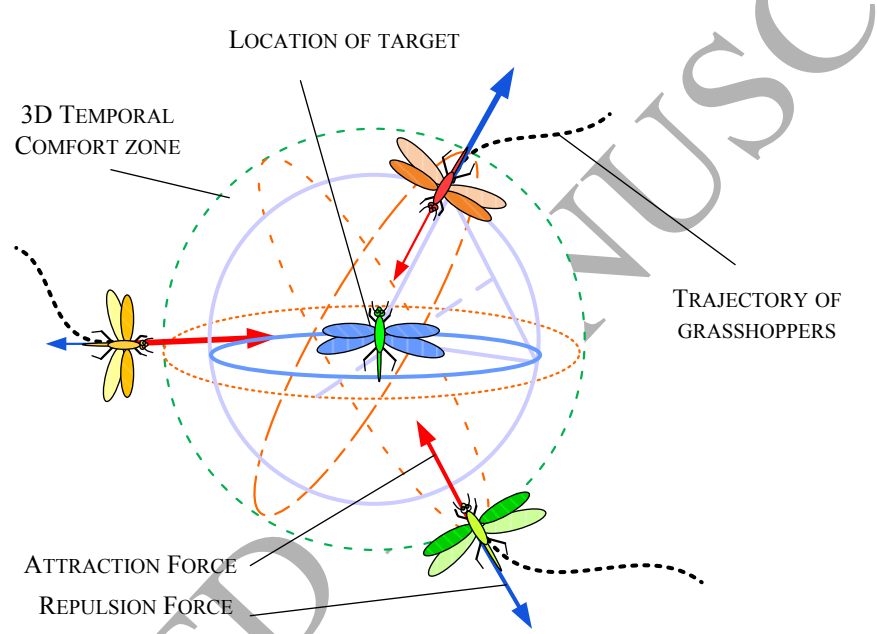

Figure 3: Primitive corrective patterns between individuals in a swarm of grasshoppers.

where $g$ denotes the gravitational constant and $\widehat{e_{g}}$ is a unity vector in the vertical direction of the surface.

The wind advection $A_{i}$ (third component in Eq. (5)) is calculated as follows:

$$
A_{i}=u \times \widehat{e_{w}}
$$

where $\mu$ represents a constant drift and $\widehat{e_{w}}$ denotes a unity vector in accordance with the wind.

In stochastic optimisation, a metaheuristic optimizer must make a fine balance between the exploration and exploitation when conducting the search to find a accurate approximation of the global optimum. Therefore, the mathematical formulation of the GOA, which was presented in Eq. (1), 
should be equipped with special parameters to achieve to this purpose. The mathematical model proposed by Saremi et al. in this regard is as follows:

$$
X_{i}^{d}=c\left(\sum_{j=1, j \neq i}^{N} c \frac{u b_{d}-l b_{d}}{2} s\left(\left|x_{j}^{d}-x_{i}^{d}\right|\right) \frac{x_{j}-x_{i}}{d_{i j}}\right)+\widehat{T}_{d}
$$

where $u b_{d}$ and $l b_{d}$ are respectively the upper bound and lower bounds in the $D^{\text {th }}$ dimension, $\widehat{T_{d}}$ is the value of the $D^{\text {th }}$ dimension of the target grasshopper. Parameter $c$ is a decreasing coefficient to shrink the comfort zone, attraction, and repulsion regions. Note that $S$ is similar to the $s$ function in Eq. (2). In Eq. (6), gravity force has been considered equal to Zero (no $G$ component), and the wind force ( $A$ component) is always towards the target grasshopper $\widehat{T_{d}}$. The adaptive parameter $c$ is considered as decreasing coefficient, it has been used twice to simulate the deceleration of grasshoppers approaching the source of food and eventually consuming it. The outer $c$ (first $c$ from the left) has been used to reduce the search coverage toward the target grasshopper as the iteration count increases, while the inner $c$ has been used to reduce the effect of the attraction and repulsion forces between grasshoppers with regard to the number of iterations to shrink the comfort, repulsion, and attraction areas.

The parameter $c$ is updated with the following relation, it should be inversely proportional to the number of executed iterations. This mechanism increases the degree of exploitation as the iteration count increases. It also reduces the comfort zone proportional to the number of iterations.

$$
c=c M a x-l \frac{c M a x-c M i n}{L}
$$

where $c M a x$ and $c M i n$ are respectively the maximum and minimum values of parameter $c, l$ is iteration, and $L$ is the maximum bound of iterations. In [76], the authors used 0.00001 and 1 for $c$ Min and $c$ Max, respectively.

\section{Binary GOA (BGOA) for feature selection}

Finding a minimal feature set has been described as a NP-hard problem [79]. Searching the best combination of features is a challenging problem especially in the wrapper-based methods. Hence, an intelligent optimization method is required to reduce the number of evaluations. 
As reported in the literature [76, 47], the GOA algorithm can reveal a superior efficacy in tackling various optimization cases. The merits of GOA motivated us to propose a binary version of the GOA optimizer and use it as the core search engine in this paper when solving FS problems [79]. Based on the NP-hard nature of FS problems, where the search space can be represented by binary values, some operators of the GOA algorithm need to be modified. In the continuous GOA, each individual updates its position based on its current position, the position of the best grasshopper found so far (target), and the position of all other grasshoppers as in Eq. (6). This behavior of the GOA is similar to other swarm-based techniques (e.g., PSO). In the GOA, the first term of Eq. (6) is analogous to the velocity vector (step) in the PSO. According to the claims provided by Mirjalili and Lewis [80], one of the easiest ways to convert an algorithm from continuous to binary version without modifying its structure is to utilize transfer functions. In the proposed approach, the transfer function (see Fig. 4) use the first term in Eq. (6), that is re-defined $\Delta X$ in Eq. (8) as the probability for changing of the position elements.

$$
\Delta X=c\left(\sum_{j=1, j \neq i}^{N} c \frac{u b_{d}-l b_{d}}{2} s\left(\left|x_{j}^{d}-x_{i}^{d}\right|\right) \frac{x_{j}-x_{i}}{d_{i j}}\right)
$$

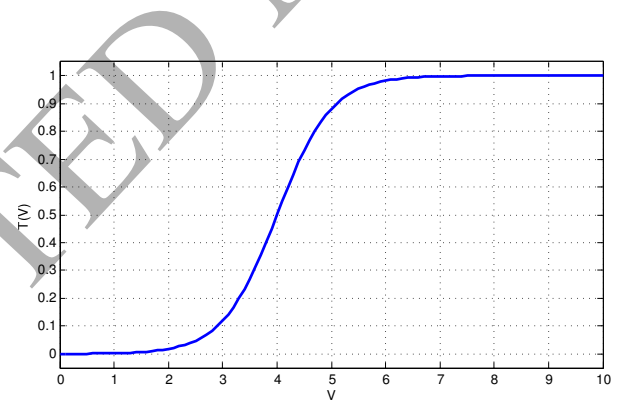

Figure 4: Sigmoidal Transfer function.

Sigmoidal function is a common transfer function proposed by Kennedy and Eberhart [81] as Eq. (9):

$$
T\left(\Delta X_{t}\right)=\frac{1}{1+e^{-\Delta X_{t}}}
$$

where $\Delta X$ represents the step vector of a search agent at a specific iteration. 
The position of the current grasshopper will be updated as expressed in Eq. (10) based on the probability value $T\left(\Delta X_{t}\right)$ obtained from Eq. (9).

$$
X_{t+1}^{k}(t+1)= \begin{cases}1 & \text { If } \text { rand }<T\left(\Delta X_{t+1}\right) \\ 0 & \text { If } \text { rand } \geq T\left(\Delta X_{t+1}\right)\end{cases}
$$

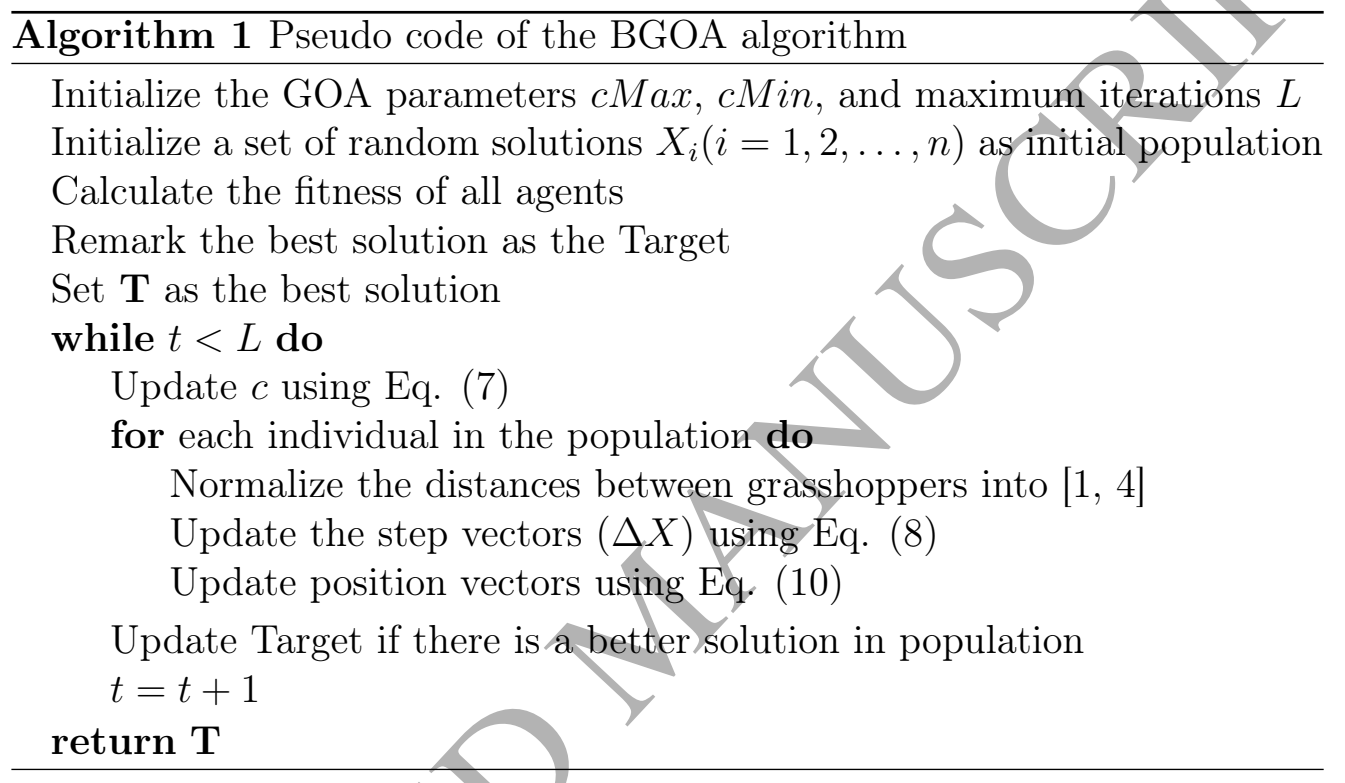

In the wrapper FS, a learning algorithm should be involved in the evaluation of the selected feature subset. In this work, the $k$-Nearest Neighbor $(k-\mathrm{NN})$ classifier [82] is utilized to attain the classification accuracy of the solution. The higher classification accuracies show that the relevant solution is better. Moreover, since the aim of FS is to eliminate the number of selected features, the smaller the number of features in the solution, the better the solution is. These are two contradictory objectives that should be taken into consideration when designing an objective function for FS algorithms. In this work, the fitness function in Eq. (11) that can balance among the selected features in each agent (minimum) and the accuracy of classification (maximum) is used to evaluate the selected subsets in all approaches.

$$
\text { Fitness }=\alpha \gamma_{R}(D)+\beta \frac{|R|}{|N|}
$$

where $\gamma_{R}(D)$ is the classification error rate of the known classier, $|R|$ is the 
number of selected features and $|N|$ is the number of features, $\alpha$ and $\beta$ are two parameters to reflect the role of classification rate and length of subset, $\alpha \in[0,1]$ and $\beta=(1-\alpha)$ adopted from [63].

\subsection{Applying the EPD strategy to BGOA}

As discussed earlier, the EPD eliminates the worst solutions from the population and replaces them by generating neighbor solutions around the good ones. The EPD mechanism is a simple but effective operator for populationbased techniques [70], therefore, it is applied to the conventional GOA here since it is also a stochastic population-based optimizer. To equip the GOA algorithm with the EPD technique, the swarm of grasshoppers is divided into two parts after sorting it based on the fitness values. The half of the worst grasshoppers is eliminated and reinitialized based on four different strategies depending on the good half of the population.

In this paper, four different strategies are utilized to combine the EPD scheme with the binary BGOA. These versions can be categorized into two main classes based on the implemented selection operators.

\subsubsection{BGOA_EPD with random selection operator}

The first model for hybridizing is to us random selection operator. For this purpose, one solution among the best three grasshoppers from the population is selected in addition to a random grasshopper. Then, the leader of 'poor' solution will be selected randomly. To implement this idea, two different approaches are designed that work based on the random selection technique:

1. BGOA_EPD: it is the simplest hybrid form of the EPD and BGOA algorithms. In this approach, the random selection mechanism is employed to select the solutions. This method also uses a simple mutation operator.

In the first approach, the top three individuals are selected and a fourth solution is generated randomly. Each solution in the worst half is repositioned around any of these four solutions depending on a random number. The process is straightforward; a random number is generated $X_{r}$ in each iteration and then one of the following four choices will be applied for repositioning of the poor solution: when $X_{r} \in[0$, $0.25]$, then the best solution is used, when $X_{r} \in[0.25,0.5]$, then the second best solution is used, when $X_{r} \in[0.5,0.75]$, then the third best solution is employed, and when $X_{r} \in[0.75,1]$, a random solution is used. 


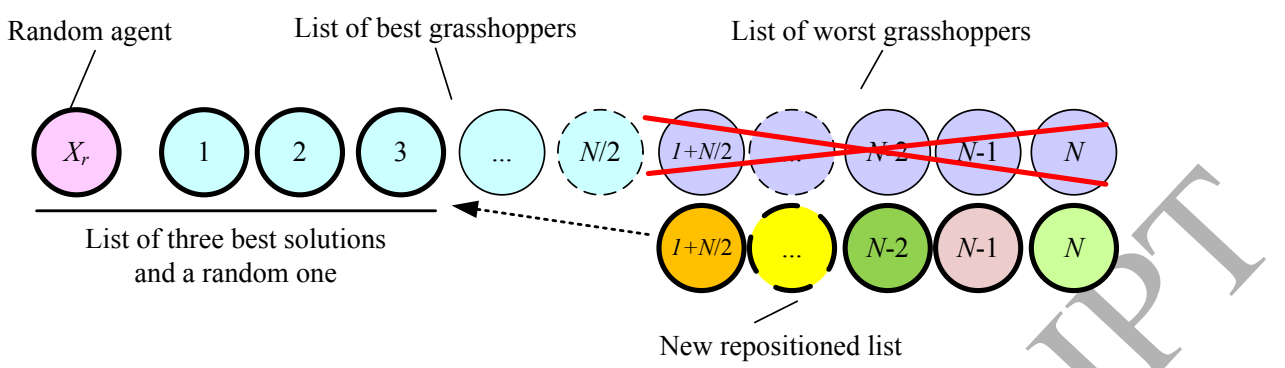

Figure 5: The sorted population and related agents that should be repositioned around the best solutions and a random one

The selected solution will be used as a starting point to reposition the poor solution. Repositioning the poor solutions around the best solutions aims to heighten the median of the swarm in each step. However, this process may cause a premature convergence of the algorithm. As a remedy, a randomly generated solution is used in the first rule to promote exploration and prevent trapping in local optima.

2. BGOA_EPD_CM: this version is similar to the BGOA_EPD and the only difference is that it also uses a crossover and a mutation operator. In the second approach, arandom number is generated and one solution is selected similar to the first strategy, then the selected solution is mutated to improve the exploration tendency of the algorithm. The mutated solution is then crossover with the poor solution.

\subsubsection{BGOA_EPD with special selection operator}

According to the findings of Talbi [27], "it does not mean that using better solutions as initial solutions will always lead to better local optima"[27]. The best individuals may bias the searching process and this may cause a premature convergence and a loss of diversity. For this reason, instead of selecting one of the three best solutions like the previous versions, two well-regarded selection mechanism are applied to select a solution from the first half of the population. For each solution in the second half of the population, select a solution from the first half using the selection mechanism; mutate and then crossover it with the poor solution. In this regard, the alternative method for hybridizing the EPD with the BGOA in the BGOAEPD algorithm is to also employ a special selection mechanism. There are two well-known selection techniques: Roulette wheel selection (RWS) [83] and the TS [84]. These methods are utilized in this work. Therefore, another 
two different strategies that can be developed for the BGOA_EPD are:

1. BGOA_EPD_Tour: in this version, a solution from the first half of the swarm is selected using the TS operator, then the same crossover and mutation operators utilized in the BGOA_EPD version are applied on the obtained solution.

The TS is the most popular selection mechanism used with GA due to its efficiency and simple implementation. In TS, a set of $n$ individuals are randomly selected from the whole population, then the best individual among the selected individuals will be selected to reposition the poor solution. The number of selected individuals called/tournament size $T s$. The advantage of $T S$ is that it gives a chance to all individuals to guide the poor solutions, which preserve the diversity of the BGOA_EPD_Tour algorithm. An example of the TS mechanism for the BGOA_EPD_Tour is illustrated in Fig.6, where three individuals are selected $(T s=3)$ and the best solution among them is picked out.

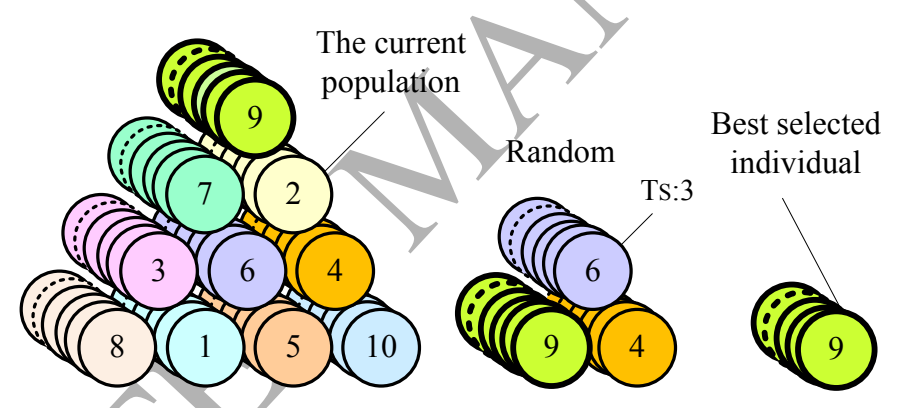

Figure 6: The mechanism of TS

After applying the TS operator, the mutation operator with suitable mutation rate is applied the selected grasshopper hoping to find a better solution in the neighbor of the selected solution and to avoid the BGOA_EPD_Tour algorithm from the premature convergence. After that, the poor solution is repositioned around the resulting solution by applying a crossover operator. In the BGOA_EPD_Tour, a solution is selected using the TS to give a chance to the lower fitness solution in the first half of the population to be selected.

2. BGOA_EPD_RWS: This version is similar to the BGOA_EPD_Tour version and the only difference is that it uses the RWS operator instead of the TS operator. 
In the BGOA_EPD_RWS, in each iteration of the BGOA process, for each solution in the worst half of the population, a solution from the first half is selected using the RWS operator. In RWS, individuals are selected with a probability based on their fitness values. In this selection strategy, a roulette wheel is formulated with a circumference equals the sum of all fitness values of the individuals (see Fig 7).
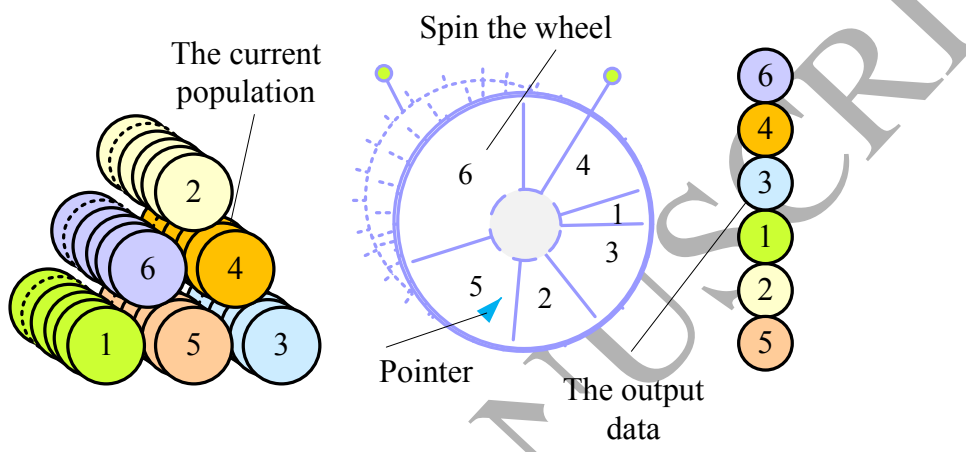

Figure 7: Selection strategy with roulette wheel mechanism

Each individual will have a segment with a size proportional to its fitness. The probability to select an individual can be seen as spinning a roulette wheel, and the segment where the pointer stops is taken and the corresponding individual will be selected. Obviously, the individuals with the largest fitness (i.e. largest segment sizes) have higher probability of being selected than those who have lower probability (i.e. smallest segment sizes). The advantage of RWS that it does not ignore any individual in the population, therefore, it preserves the diversity of the population.

After selecting a solution using the RWS operator, it is mutated to explore more regions of the feature space, then; the resulted solution from the mutation operator is used to reposition a solution from the second half by applying a crossover operator.

The mutation rate $r$ for all related approaches is shown in Eq. (12). The parameter $r$ is decremented from 0.9 to 0,linearly, according to the iteration number $i$.

$$
r=0.9\left(1+\frac{(1-i)}{L-1}\right)
$$

where $L$ was the maximum number of iterations. 
The main difference between these versions is that they use different selection operators. In addition, the BGOA_EPD_CM uses the best solutions in the population while BGOA_EPD_RWS and BGOA_EPD_Tour variants do not use this policy. They use other solutions from the first half of the population.

The overall pseudo code of the BGOA_EPD algorithm is described in Algorithm 2. Flowchart of the BGOA_EPD is also demonstrated in Fig. 8.

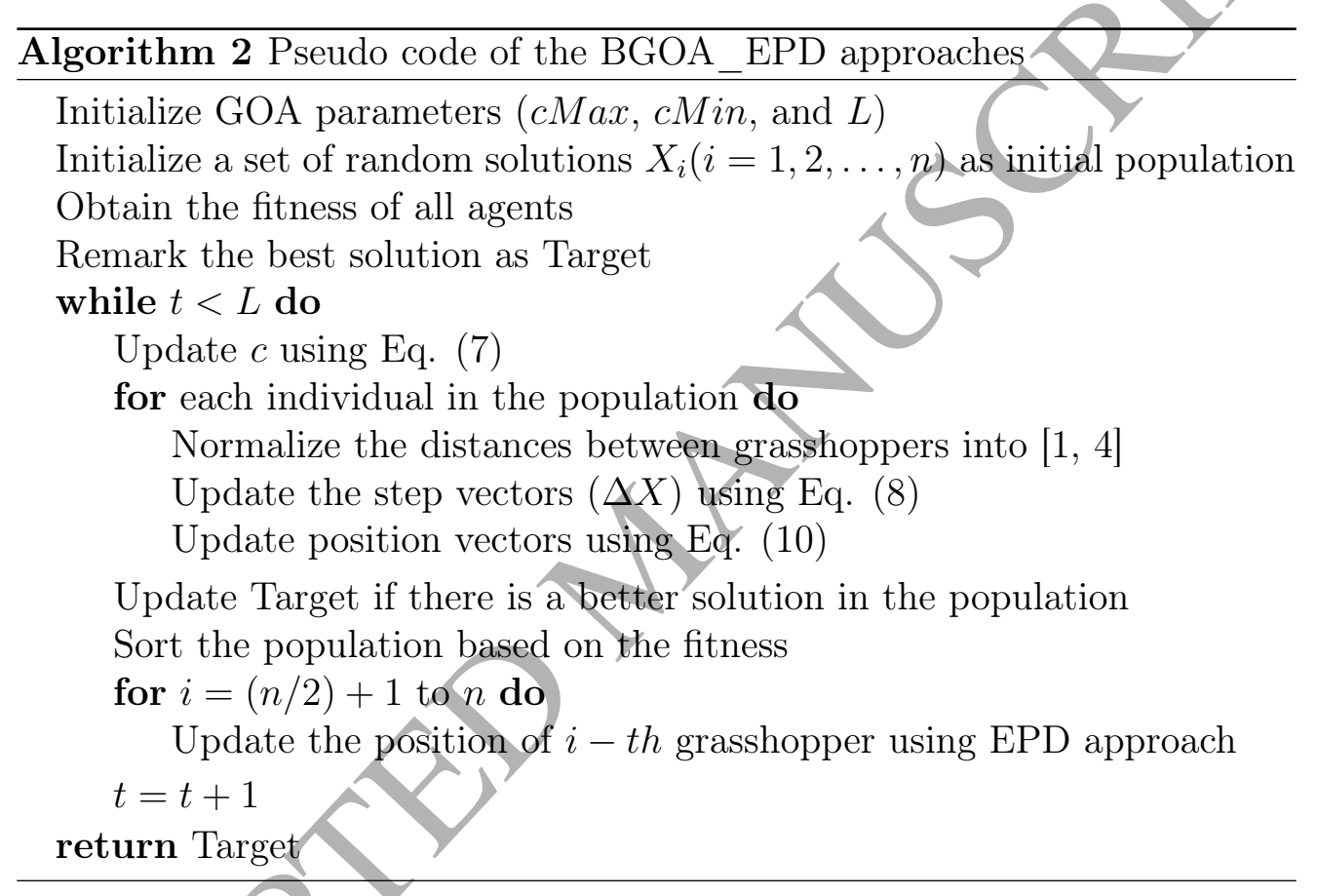




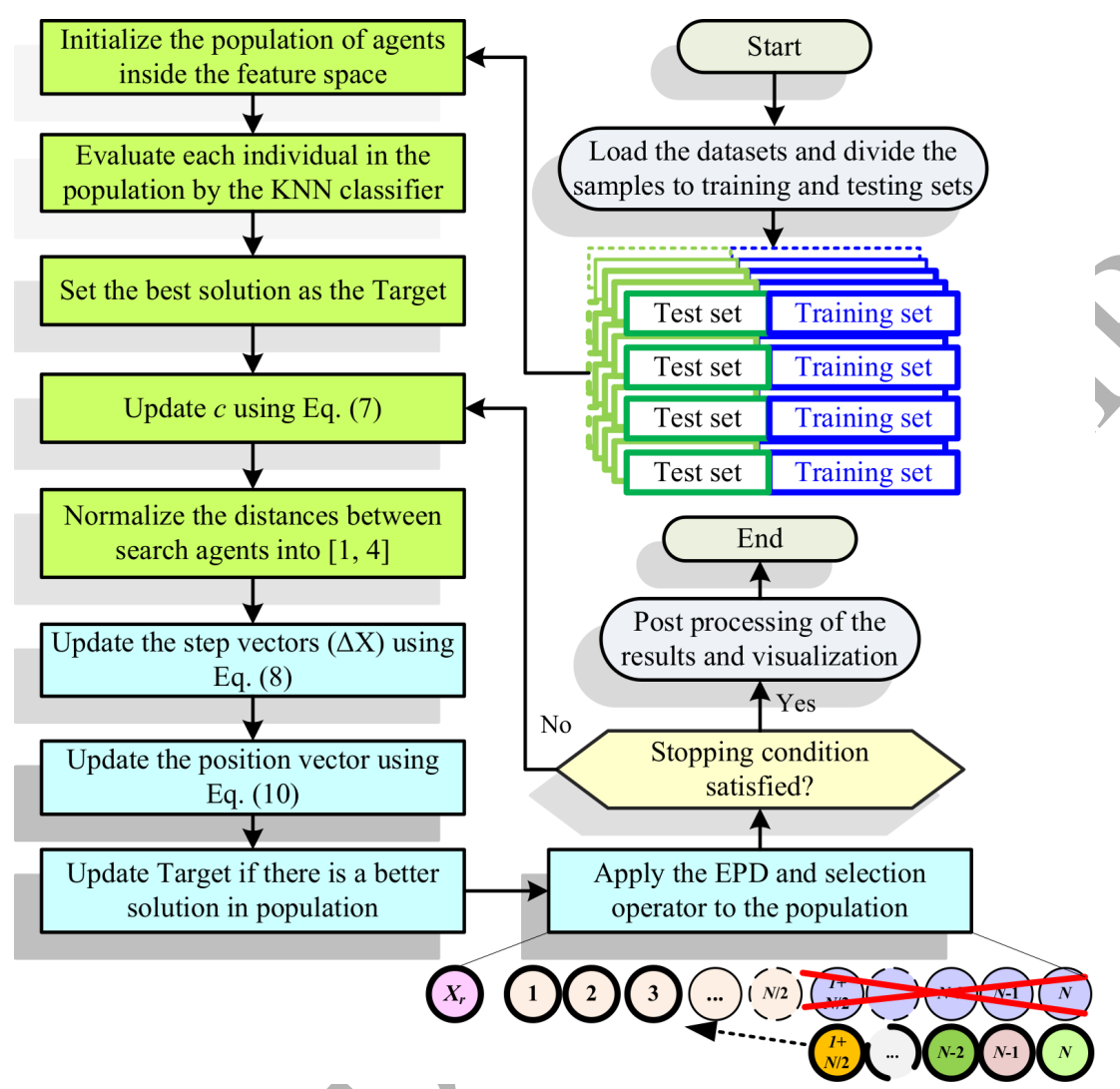

Figure 8: The overall steps for proposed BGOA_EPD approach

Note that the computational complexity of the proposed BGOA_EPD is not significantly different from the GOA. The computational complexity of GOA is of $O\left(t \times d \times n^{2}\right)$ where $t$ indicates the number of iterations, $d$ is the number of variables, and $n$ shows the number of solutions. The proposed binary operators do not change the computational complexity since they have been applied to the position updating mechanism of the original GOA. To re-initialize $50 \%$ of solutions, however, the additional complexity of $O(n / 2)$ is required, so the overall computational complexity of the proposed BGOA_EPD is $O\left(t \times d \times n^{2}+n / 2\right)$. Note that due to the need to re-evaluate the objective value of half of the solutions, the number of function evaluations in BGOA_EPD is $n / 2$ units more that of GOA. 


\section{Experimental results and discussions}

In this section, the efficacy of the proposed hybrid EPD_BGOA versions in dealing with 22 well-regarded datasets including Exactly, Breastcancer, Zoo, IonosphereEW, Vote, WaveformEW, WineEW, HeartEW, Colon, Clean1, M-of-n, Tic-tac-toe, KrvskpEW, Leukemia, and SonarEW is investigated.

Table 2 reports the brief description of the 22 datasets utilized. For more details, interested readers are referred to the UCI source. These benchmark cases have been studied in several well-established works. The utilized test set cover different traits and the instances of small to high dimensional datasets and can examine the searching competencies of EA and metaheuristics in tackling the FS problems. Different variants of BGOA algorithm were employed to search for the best reduct with the minimum error rate based on KNN classifier (where $K=5$ [85]) with the Euclidean distance metric. For evaluation purposes, each datasets is divided into training and testing sets where $80 \%$ of the instances in the datasets were used for training purposes and the rest of them is utilized for testing tasks [86].

All the fair tests and the computed results in this research are conducted and prepared on a PC with Intel Core(TM) i5-5200U 2.2GHz CPU and 4.0GB RAM. The maximum iterations ( $L$ ) is set to 100 and the number of search agents $(N)$ is 10. Additionally, all statistical results are recorded over 30 independent runs. The dimension of cases is equal to the number of features in each experimented dataset. The $\alpha$ and $\beta$ parameters in the fitness equation are set to 0.99 and 0.01 , respectively. 
Table 2: List of used datasets

\begin{tabular}{llcc}
\hline No. & Dataset & No. of Features & No. of instances \\
\hline 1. & Breastcancer & 9 & 699 \\
2. & BreastEW & 30 & 596 \\
3. & Exactly & 13 & 1000 \\
4. & Exactly2 & 13 & 1000 \\
5. & HeartEW & 13 & 270 \\
6. & Lymphography & 18 & 148 \\
7. & M-of-n & 13 & 1000 \\
8. & PenglungEW & 325 & 73 \\
9. & SonarEW & 60 & 208 \\
10. & SpectEW & 22 & 267 \\
11. & CongressEW & 16 & 435 \\
12. & IonosphereEW & 34 & 351 \\
13. & KrvskpEW & 36 & 3196 \\
14. & Tic-tac-toe & 9 & 958 \\
15. & Vote & 16 & 300 \\
16. & WaveformEW & 40 & 5000 \\
17. & WineEW & 13 & 178 \\
18. & Zoo & 16 & 101 \\
19. & Clean1 & 166 & 476 \\
20. & Semeion & 265 & 1593 \\
21. & Colon & 2000 & 62 \\
22. & Leukemia & 7129 & 72 \\
\hline & & &
\end{tabular}

\subsection{Evaluation of proposed methods}

In this part, the efficiency, convergence and the quality of the results of four developed hybrid approaches are deeply measured and compared to each other's to distinguish the preeminent variant for more advanced investigations. The four techniques utilizing different operators and the random, TS and RWS mechanisms are substantiated and compared to judge and discover the influence of the crossover and mutation strategies and using a specific selection scheme in preference to the random selection policy on either the results or efficacy of the proposed variants. The performance of the proposed optimizers is evaluated and compared in terms of the average classification accuracy (Acc), selection size, and fitness values, computational times, and convergence rates over all runs of each technique. The Acc is measured via the nominated features on the used dataset. The standard deviation (StdDev) of all proposed versions is also provided for all metrics, datasets and algorithms.

Table 3 exposes the attained Acc and related StdDev results for the BGOA_S algorithm versus other designed versions. Tables 4-6 also reflect the average selected attributes, fitness, and CPU time values along with the related StdDev for the proposed techniques.

From Table 3, it can be detected that the hybrid BGOA_EPD_Tour can 
Table 3: Comparison of the BGOA_S with four hybrid versions using Acc and StdDev metrics

\begin{tabular}{|c|c|c|c|c|c|c|c|c|c|c|}
\hline \multirow{2}{*}{ Dataset } & \multicolumn{2}{|c|}{ BGOA_S } & \multicolumn{2}{|c|}{ BGOA_EPD } & \multicolumn{2}{|c|}{ BGOA_EPD_CM } & \multicolumn{2}{|c|}{ BGOA_EPD_RWS } & \multicolumn{2}{|c|}{ BGOA_EPD_Tour } \\
\hline & Acc & StdDev & Acc & StdDev & Acc & StdDev & Acc & StdDev & Acc & StdDev \\
\hline Breastcancer & 0.969 & 0.000 & 0.966 & 0.000 & 0.969 & 0.000 & 0.977 & 0.000 & 0.980 & 0.001 \\
\hline BreastEW & 0.960 & 0.005 & 0.962 & 0.002 & 0.963 & 0.003 & 0.964 & 0.003 & 0.947 & 0.005 \\
\hline Exactly & 0.946 & 0.036 & 0.997 & 0.006 & 0.993 & 0.009 & 0.999 & 0.008 & 0.999 & 0.005 \\
\hline Exactly2 & 0.760 & 0.000 & 0.734 & 0.005 & 0.744 & 0.013 & 0.762 & 0.000 & 0.780 & \\
\hline HeartEW & 0.826 & 0.010 & 0.842 & 0.006 & 0.841 & 0.012 & 0.815 & 0.008 & 0.833 & 0.004 \\
\hline Lymphography & 0.815 & 0.012 & 0.895 & 0.013 & 0.844 & 0.012 & 0.878 & 0.009 & 0.868 & \\
\hline M-of-n & 0.979 & 0.030 & 0.997 & 0.007 & 0.999 & 0.004 & 0.999 & 0.004 & 1.000 & $0.000^{\circ}$ \\
\hline PenglungEW & 0.861 & 0.015 & 0.868 & 0.008 & 0.839 & 0.021 & 0.750 & 0.012 & 0.927 & 0.013 \\
\hline SonarEW & 0.895 & 0.011 & 0.921 & 0.007 & 0.883 & 0.006 & 0.922 & 0.008 & 0.912 & 0.009 \\
\hline SpectEW & 0.851 & 0.011 & 0.850 & 0.007 & 0.882 & 0.006 & 0.852 & 0.007 & 0.826 & 0.010 \\
\hline CongressEW & 0.953 & 0.004 & 0.983 & 0.003 & 0.974 & 0.004 & 0.983 & 0.004 & 0.964 & 0.005 \\
\hline IonosphereEW & 0.883 & 0.007 & 0.907 & 0.004 & 0.922 & 0.007 & 0.911 & & 0.899 & 0.007 \\
\hline KrvskpEW & 0.956 & 0.008 & 0.960 & 0.006 & 0.959 & 0.006 & 0.963 & 0.004 & 0.968 & 0.003 \\
\hline Tic-tac-toe & 0.803 & 0.007 & 0.797 & 0.001 & 0.789 & 0.000 & 0.785 & 0.000 & 0.808 & 0.000 \\
\hline Vote & 0.951 & 0.004 & 0.948 & 0.006 & 0.953 & 0.004 & 0.960 & 0.003 & 0.966 & 0.003 \\
\hline WaveformEW & 0.729 & 0.009 & 0.739 & 0.006 & 0.743 & 0.004 & 0.740 & 0.004 & 0.737 & 0.003 \\
\hline WineEW & 0.979 & 0.004 & 0.994 & 0.006 & 0.993 & 0.005 & 0.988 & 0.003 & 0.989 & 0.000 \\
\hline Zoo & 0.990 & 0.010 & 0.976 & 0.008 & 0.960 & 0.004 & 1.000 & 0.000 & 0.993 & 0.009 \\
\hline Clean1 & 0.883 & 0.008 & 0.885 & 0.005 & 0.893 & 0.005 & 0.885 & 0.006 & 0.863 & 0.004 \\
\hline Semeion & 0.975 & 0.002 & 0.979 & 0.001 & 0.986 & 0.001 & 0.974 & 0.001 & 0.976 & 0.002 \\
\hline Colon & 0.745 & 0.010 & 0.812 & 0.012 & 0.712 & 0.008 & 0.810 & 0.010 & 0.870 & 0.006 \\
\hline Leukemia & 0.928 & 0.014 & 0.889 & 0.000 & 0.931 & 0.014 & 0.855 & 0.012 & 0.931 & 0.014 \\
\hline
\end{tabular}

relatively outperform other competitors in terms of Acc and StdDev metrics in dealing with 10 and 11 datasets, respectively. The simple binary BGOA_S cannot reveal higher accuracies than any hybrid variant over all 22 datasets. For the M-of-n dataset, the BGOA_EPD_Tour has classified with $100 \%$ Acc and 0 StdDev using only 6.47 attributes. From Table 3 , it is observed that the BGOA_EPD_RWS can provide superior Acc rates compared to other varieties in tackling the BreastEW, CongressEW, SonarEW, and Zoo datasets. The BGOA_EPD_RWS has attained the Acc of $100 \%$ in solving the Zoo test case. The BGOA_EPD_CM outperform others in terms of Acc in dealing with the 6 datasets: IonosphereEW, SpectEW, WaveformEW, Clean1, Semeion, and Leukemia problems. The BGOA_EPD has outperformed competitors in realizing the HeartEW, Lymphography, and WineEW. In tackling the CongressEW, both BGOA_EPD and BGOA_EPD_RWS have reached to a same Acc rate $98.3 \%$, while based on selected attributes in Table 4, the BGOA_EPD_RWS with 5.5 selected attributes has outperformed the BGOA EPD.

Regarding the Acc rates, the BGOA_EPD_Tour can outperform the BGOA_S over 19 problems and there is a marked shift in the rates for the BGOA_EPD_Tour and improvement varies from 1\% to 12.5\%.A comparable 
pattern can be detected from the results of BGOA_EPD_RWS, it outperform the BGOA_S technique on 17 datasets, whereas the BGOA_EPD_CM and BGOA_EPD outperform the basic optimizer on 15 problems.

According to the selected attributes (Atts) in Table 4, it is seen that the simple BGOA_S is better than the BGOA_EPD_Tour on 19 datasets. It also is superior to the BGOA_EPD_RWS and BGOA_EPD_CM in dealing with 17 problems.

Table 4: Average selected attributes using the developed algorithms

\begin{tabular}{|c|c|c|c|c|c|c|c|c|c|c|}
\hline \multirow{2}{*}{ Dataset } & \multicolumn{2}{|c|}{ BGOA_S } & \multicolumn{2}{|c|}{ BGOA_EPD } & \multicolumn{2}{|c|}{ BGOA_EPD_CM } & \multicolumn{2}{|c|}{ BGOA_EPD_RWS } & \multicolumn{2}{|c|}{ BGOA_EPD_Tour } \\
\hline & Atts & StdDev & Atts & StdDev & Atts & StdDev & Atts & StdDev & Atts & StdDev \\
\hline Breastcancer & 4.00 & 0.000 & 5.13 & 0.346 & 4.17 & 0.379 & 4.73 & 0.980 & 5.00 & 0.000 \\
\hline BreastEW & 15.37 & 2.697 & 20.00 & 2.729 & 17.50 & 1.889 & 17,20 & 2.747 & 17.33 & 2.440 \\
\hline Exactly & 7.63 & 0.809 & 6.60 & 0.498 & 6.57 & 0.504 & 6.43 & 0.568 & 6.53 & 0.571 \\
\hline Exactly2 & 1.27 & 0.450 & 7.97 & 0.809 & 5.67 & 3.565 & 1.50 & 0.509 & 1.53 & 0.507 \\
\hline HeartEW & 6.77 & 1.524 & 6.67 & 0.922 & 5.77 & 0.817 & 6.13 & 1.548 & 8.40 & 1.037 \\
\hline Lymphography & 7.47 & 2.080 & 10.60 & 1.003 & 10.60 & 1.0 & 1.60 & 1.476 & 10.63 & 1.217 \\
\hline M-of-n & 7.53 & 0.973 & 6.53 & 0.629 & 6.57 & 0.504 & 6.57 & 0.568 & 6.47 & 0.507 \\
\hline PenglungEW & 150.13 & 8.509 & 166.53 & 15.937 & 198.90 & 8.707 & 174.77 & 15.460 & 178.33 & 15.486 \\
\hline SonarEW & 28.57 & 3.191 & 36.37 & 3.157 & 36.03 & 3.489 & 35.73 & 2.778 & 36.77 & 4.240 \\
\hline SpectEW & 9.93 & 1.856 & 14.13 & 1.995 & 12.83 & 2.437 & 11.93 & 2.463 & 11.10 & 3.044 \\
\hline CongressEW & 4.33 & 1.322 & 7.67 & 1.729 & 6.53 & $2.145^{\prime}$ & 5.50 & 1.592 & 5.77 & 2.012 \\
\hline IonosphereEW & 13.43 & 3.115 & 18.93 & 3.269 & 17.93 & 2.420 & 17.77 & 3.785 & 16.40 & 3.701 \\
\hline KrvskpEW & 19.90 & 3.010 & 22.33 & 3.010 & 22.10 & 2.551 & 22.43 & 2.417 & 21.67 & 2.496 \\
\hline Tic-tac-toe & 6.83 & 0.379 & 5.03 & 0.183 & 6.00 & 0.000 & 6.10 & 0.305 & 5.00 & 0.000 \\
\hline Vote & 5.27 & 2.083 & 6.73 & 1.015 & 6.57 & 1.995 & 6.07 & 1.617 & 5.43 & 1.223 \\
\hline WaveformEW & 21.20 & 2.952 & 25.53 & 3.082 & 25.80 & 3.295 & 24.93 & 2.876 & 26.23 & 3.451 \\
\hline WineEW & 6.33 & 1.348 & 7.27 & 0.944 & 7.23 & 0.817 & 6.83 & 1.147 & 8.80 & 1.472 \\
\hline Zoo & 8.13 & 1.167 & 7.97 & 1.426 & 7.93 & 1.230 & 7.77 & 0.774 & 9.17 & 1.967 \\
\hline clean1 & 82.93 & 5.948 & 103.80 & 6.880 & 105.20 & 6.206 & 96.00 & 9.127 & 92.60 & 7.802 \\
\hline Semeion & 134.90 & 7.662 & 169.63 & 11.137 & 171.50 & 7.361 & 159.87 & 9.885 & 157.03 & 11.485 \\
\hline Colon & 967.87 & 23.882 & 1194.33 & 89.430 & 1198.33 & 97.070 & 1050.83 & 70.247 & 1063.67 & 64.618 \\
\hline Leukemia & 3495.23 & 48.699 & 4138.27 & 373.767 & 4366.87 & 288.129 & 3896.63 & 292.866 & 3768.80 & 224.842 \\
\hline
\end{tabular}

Inspecting the fitness measures (Fitness) in Table 5, the best optimizer is the BGOA_EPD_Tour. It shows the lowest values for the objective function in tackling the 10 datasets: Breastcancer, Exactly, Exactly2, KrvskpEW, M-of-n, PenglungEW, Tic-tac-toe, Vote, Colon, and Leukemia. The BGOA_EPD_RWS has shown a relatively good performance in dealing with 4 test cases: BreastEW, CongressEW, SonarEW, and Zoo. The BGOA_EPD_CM has provided a lower fitness for IonosphereEW, SpectEW, WaveformEW, Clean1, and Semeion datasets.

From Table 6, it can be observed that the BGOA_S is the fastest approach in a same computational environment with other optimizers. When comparing the algorithms with the TS-based and RWS-based selection operators, it is seen that for 19 datasets, BGOA_EPD_RWS outperforms the BGOA_EPD_Tour. For only BreastEW, Exactly, and Tic-tac-toe, the 
Table 5: Average fitness values for proposed versions

\begin{tabular}{|c|c|c|c|c|c|c|c|c|c|c|}
\hline \multirow{2}{*}{ Dataset } & \multicolumn{2}{|c|}{ BGOA_S } & \multicolumn{2}{|c|}{ BGOA_EPD } & \multicolumn{2}{|c|}{ BGOA_EPD_CM } & \multicolumn{2}{|c|}{ BGOA_EPD_RWS } & \multicolumn{2}{|c|}{ BGOA_EPD_Tour } \\
\hline & Fitness & StdDev & Fitness & StdDev & Fitness & StdDev & Fitness & StdDev & Fitness & StdDev \\
\hline Breastcancer & 0.036 & 0.000 & 0.040 & 0.000 & 0.036 & 0.000 & 0.028 & 0.001 & 0.026 & 0.001 \\
\hline BreastEW & 0.045 & 0.005 & 0.044 & 0.002 & 0.043 & 0.003 & 0.041 & 0.003 & 0.058 & 0.00 \\
\hline Exactly & 0.059 & 0.036 & 0.008 & 0.006 & 0.012 & 0.010 & 0.006 & 0.008 & 0.006 & 0.006 \\
\hline Exactly2 & 0.239 & 0.000 & 0.269 & 0.005 & 0.257 & 0.015 & 0.237 & 0.000 & 0.219 & 0.000 \\
\hline HeartEW & 0.178 & 0.009 & 0.161 & 0.006 & 0.162 & 0.012 & 0.188 & 0.008 & 0.171 & \\
\hline Lymphography & 0.187 & 0.012 & 0.110 & 0.013 & 0.161 & 0.012 & 0.127 & 0.009 & 0.137 & 0.011 \\
\hline M-of-n & 0.027 & 0.030 & 0.008 & 0.007 & 0.006 & 0.004 & 0.006 & 0.004 & 0.005 & 0.000 \\
\hline PenglungEW & 0.142 & 0.015 & 0.136 & 0.008 & 0.166 & 0.020 & 0.253 & 0.012 & 0.078 & 0.012 \\
\hline SonarEW & 0.109 & 0.010 & 0.084 & 0.007 & 0.122 & 0.006 & 0.083 & 0.008 & 0.094 & 0.008 \\
\hline SpectEW & 0.152 & 0.011 & 0.155 & 0.007 & 0.123 & 0.006 & 0.152 & 0.006 & 0.177 & 0.010 \\
\hline Tic-tac-toe & 0.203 & 0.007 & 0.206 & 0.001 & 0.215 & 0.000 & 0.220 & 0.000 & 0.196 & 0.000 \\
\hline CongressEW & 0.049 & 0.004 & 0.022 & 0.003 & 0.030 & 0.004 & 0.020 & 0.005 & 0.039 & 0.005 \\
\hline IonosphereEW & 0.120 & 0.008 & 0.098 & 0.004 & 0.082 & 0.007 & 0.094 & 0.005 & 0.105 & 0.007 \\
\hline KrvskpEW & 0.049 & 0.008 & 0.046 & 0.006 & 0.047 & 0.006 & 0.043 & & 0.038 & 0.003 \\
\hline Vote & 0.052 & 0.004 & 0.055 & 0.006 & 0.050 & 0.005 & 0.044 & 0.003 & 0.037 & 0.003 \\
\hline WaveformEW & 0.274 & 0.009 & 0.265 & 0.006 & 0.261 & 0.004 & 0.263 & 0.005 & 0.267 & 0.003 \\
\hline WineEW & 0.025 & 0.004 & 0.012 & 0.005 & 0.013 & 0.005 & 0.017 & 0.003 & 0.018 & 0.001 \\
\hline Zoo & 0.015 & 0.010 & 0.028 & 0.007 & 0.044 & 0.004 & 0.005 & 0.000 & 0.012 & 0.008 \\
\hline Clean1 & 0.121 & 0.008 & 0.120 & 0.004 & 0.112 & 0.005 & 0.120 & 0.006 & 0.141 & 0.004 \\
\hline Semeion & 0.030 & 0.002 & 0.027 & 0.001 & 0.020 & 0.001 & 0.031 & 0.001 & 0.030 & 0.001 \\
\hline Colon & 0.257 & 0.010 & 0.192 & 0.012 & 0.291 & 0.008 & 0.194 & 0.010 & 0.134 & 0.006 \\
\hline Leukemia & 0.076 & 0.014 & 0.116 & 0.001 & 0.074 & 0.014 & 0.149 & 0.012 & 0.073 & 0.014 \\
\hline
\end{tabular}

BGOA_EPD_Tour has a slightly better run speed.

The average ranking of the proposed binary and hybrid versions in terms of Acc, Att, and fitness metrics is presented in Table 8. In Table 8, the rank of each method on a dataset is calculated, then, the sum of the ranks based on each metric is obtained. The total sum shows the sum of the ranks of each optimizer based on all metrics. The final rank shows the final average place of each algorithm in handling all 22 datasets. The utilized ranking system gives a lower place to those items that have better value according to a specific metric. The overall and final ranks are inside $[1,5]$ interval.

Table 8 divulges that the BGOA_EPD_RWS and BGOA_EPD_Tour has achieved to the best places. Regarding the Acc metric, the BGOA_EPD_Tour won the competition and BGOA_EPD_RWS was the second/ winner, while the BGOA_EPD_CM and BGOA_EPD were both the third front-runners and BGOA_S gained the last stage. Based on the orders for the Fit measure, the best solvers can be spotted as the BGOA_EPD_Tour, BGOA_EPD_RWS, BGOA_EPD_CM, BGOA_EPD, and $\bar{B} G O A_{-}{ }_{S}$, respectively. Regarding the Att measure, the best is the BGOA_S, while the BGOA_EPD_Tour and BGOA_EPD_RWS have acquired the second and third places.

Based on the final ranks, the best two algorithms (BGOA_EPD_RWS 
Table 6: Average CPU time (seconds) of proposed techniques

\begin{tabular}{|c|c|c|c|c|c|c|c|c|c|c|}
\hline \multirow{2}{*}{ Dataset } & \multicolumn{2}{|c|}{ BGOA_S } & \multicolumn{2}{|c|}{ BGOA_EPD } & \multicolumn{2}{|c|}{ BGOA_EPD_CM } & \multicolumn{2}{|c|}{ BGOA_EPD_RWS } & \multicolumn{2}{|c|}{ BGOA_EPD_Tour } \\
\hline & Time & StdDev & Time & StdDev & Time & StdDev & Time & StdDev & Time & StdDev \\
\hline Breastcancer & 3.537 & 0.248 & 4.053 & 0.232 & 4.370 & 0.222 & 4.131 & 0.214 & 4.146 & 0.191 \\
\hline BreastEW & 3.780 & 0.199 & 5.256 & 0.221 & 5.761 & 0.237 & 5.427 & 0.249 & 5.404 & 0.241 \\
\hline Exactly & 4.874 & 0.238 & 6.166 & 0.301 & 6.881 & 0.313 & 6.155 & 0.262 & 6.014 & 0.277 \\
\hline Exactly2 & 5.223 & 0.344 & 6.204 & 0.285 & 6.882 & 0.351 & 5.934 & 0.265 & 5.942 & 0.257 \\
\hline HeartEW & 2.803 & 0.164 & 3.276 & 0.183 & 3.667 & 0.165 & 3.484 & 0.190 & 3.505 & 0.178 \\
\hline Lymphography & 2.540 & 0.148 & 3.145 & 0.144 & 3.547 & 0.169 & 3.413 & 0.161 & 3.427 & 0.162 \\
\hline M-of-n & 4.792 & 0.234 & 5.641 & 0.220 & 6.406 & 0.288 & 5.812 & 0.279 & 6.173 & 0.271 \\
\hline penglungEW & 2.836 & 0.164 & 18.597 & 0.784 & 21.038 & 0.920 & 19.494 & 0.756 & 9.560 & 0.822 \\
\hline SonarEW & 2.713 & 0.160 & 5.469 & 0.263 & 6.113 & 0.262 & 5.774 & 0.240 & 5.801 & 0.245 \\
\hline SpectEW & 2.705 & 0.154 & 3.465 & 0.147 & 3.916 & 0.189 & 3.746 & 0.183 & 3.775 & 0.164 \\
\hline Tic-tac-toe & 4.226 & 0.210 & 4.961 & 0.210 & 5.227 & 0.255 & 5.214 & 0.250 & 5.097 & 0.222 \\
\hline CongressEW & 3.248 & 0.160 & 3.835 & 0.186 & 4.273 & 0.207 & 4.093 & 0.211 & 4.109 & 0.205 \\
\hline IonosphereEW & 2.899 & 0.152 & 4.441 & 0.205 & 4.988 & 0.207 & .717 & 0.201 & 4.735 & 0.199 \\
\hline KrvskpEW & 49.520 & 1.668 & 58.357 & 1.773 & 62.814 & 3.055 & 56.831 & 1.871 & 57.043 & 1.743 \\
\hline Vote & 2.799 & 0.158 & 3.364 & 0.164 & 3.491 & 0.154 & 3.618 & 0.187 & 3.640 & 0.179 \\
\hline WaveformEW & 123.546 & 4.381 & 152.292 & 4.186 & 157.271 & 5.641 & 145.890 & 4.420 & 152.167 & 4.812 \\
\hline WineEW & 2.492 & 0.151 & 3.054 & 0.152 & 3.176 & 0.152 & 3.281 & 0.176 & 3.766 & 0.301 \\
\hline Zoo & 2.485 & 0.145 & 3.076 & 0.137 & 3.182 & 0.156 & 3.371 & 0.186 & 3.614 & 0.240 \\
\hline clean1 & 7.204 & 0.225 & 16.423 & 0.542 & 16.988 & 0.566 & 16.904 & 0.600 & 17.220 & 0.608 \\
\hline Semeion & 88.934 & 1.514 & 129.229 & 2.182 & 130.251 & 2.154 & 120.308 & 2.480 & 123.254 & 2.147 \\
\hline Colon & 4.964 & 0.338 & 100.832 & 3.628 & 106.310 & 4.461 & 107.901 & 4.333 & 112.236 & 4.364 \\
\hline Leukemia & 15.322 & 0.884 & 383.067 & 19.075 & 379.074 & 15.501 & 381.100 & 15.244 & 394.688 & 15.418 \\
\hline
\end{tabular}

Table 7: P-values of the Wilcoxon test the classification accuracy results of the proposed approaches ( $\mathrm{p} \geq 0.05$ are underlined, and $\mathrm{N} / \mathrm{A}$ means not applicable).

\begin{tabular}{|c|c|c|c|c|c|}
\hline & BGOA_S & BGOA_EPD & BGOA_EPD-CM & BGOA_EPD_RWS & BGOA_EPD_Tour \\
\hline Breast Cancer & $2.71 \mathrm{E}-\overline{14}$ & $2.71 \mathrm{E}-14$ & $2 . \overline{71 \mathrm{E}-14}$ & $1.22 \mathrm{E}-12$ & $\bar{N} / \mathrm{A}$ \\
\hline BreastEW & 1.42E-04 & & $2.25 \mathrm{E}-01$ & $\mathrm{~N} / \mathrm{A}$ & $1.88 \mathrm{E}-11$ \\
\hline Exactly & $2.04 \mathrm{E}-10$ & $9.53 \mathrm{E}-06$ & $\overline{1.21 \mathrm{E}-04}$ & $1.00 \mathrm{E}+00$ & $\mathrm{~N} / \mathrm{A}$ \\
\hline Exactly2 & 1.69E-14 & $1.01 \mathrm{E}-12$ & $5.37 \mathrm{E}-13$ & 1.69E-14 & $\mathrm{N} / \mathrm{A}$ \\
\hline HeartEW & 7.62E-09 & $\mathrm{N} / \mathrm{A}$ & $\underline{2.15 \mathrm{E}-01}$ & $1.29 \mathrm{E}-11$ & 7.19E-08 \\
\hline Lymphography & $1.98 \mathrm{E}-11$ & $\mathrm{~N} / \mathrm{A}$ & $\overline{1.79 \mathrm{E}-11}$ & $6.41 \mathrm{E}-06$ & $1.89 \mathrm{E}-11$ \\
\hline M-of-n & 8.60E-07 & 4.19E-02 & $\underline{8.15 \mathrm{E}-02}$ & $\underline{3.34 \mathrm{E}-01}$ & $\mathrm{~N} / \mathrm{A}$ \\
\hline penglungEV & $3.75 \mathrm{E}-12$ & $8.38 \mathrm{E}-13$ & $\overline{5.90 \mathrm{E}-12}$ & $\overline{2.63 \mathrm{E}-12}$ & $\mathrm{~N} / \mathrm{A}$ \\
\hline SonarEW & $4.77 \mathrm{E}-11$ & $7.19 \mathrm{E}-01$ & $7.57 \mathrm{E}-12$ & $\mathrm{~N} / \mathrm{A}$ & 2.11E-05 \\
\hline SpectEW & $2.46 \mathrm{E}-11$ & $\overline{1.56 \mathrm{E}-11}$ & $\mathrm{~N} / \mathrm{A}$ & $1.29 \mathrm{E}-11$ & $1.79 \mathrm{E}-11$ \\
\hline CongressEW & $1.35 \mathrm{E}-11$ & $\underline{6.58 \mathrm{E}-01}$ & 3.87E-09 & $\mathrm{N} / \mathrm{A}$ & $1.54 \mathrm{E}-11$ \\
\hline IonosphereE & $1.82 \mathrm{E}-11$ & $2.68 \mathrm{E}-10$ & $\mathrm{~N} / \mathrm{A}$ & $2.95 \mathrm{E}-08$ & $5.21 \mathrm{E}-11$ \\
\hline KrvskpEW & 3.14E-09 & $4.23 \mathrm{E}-06$ & $1.48 \mathrm{E}-08$ & $3.29 \mathrm{E}-06$ & $\mathrm{~N} / \mathrm{A}$ \\
\hline Tic-tac-toe & $1.19 \mathrm{E}-13$ & $2.71 \mathrm{E}-14$ & $2.71 \mathrm{E}-14$ & $1.69 \mathrm{E}-14$ & $\mathrm{~N} / \mathrm{A}$ \\
\hline Vote & $9.06 \mathrm{E}-12$ & 7.00E-12 & $1.64 \mathrm{E}-11$ & $2.11 \mathrm{E}-08$ & $\mathrm{~N} / \mathrm{A}$ \\
\hline WaveformEW & $3.04 \mathrm{E}-08$ & $3.16 \mathrm{E}-03$ & $\mathrm{~N} / \mathrm{A}$ & 3.13E-02 & $3.73 \mathrm{E}-07$ \\
\hline WineEW & $1.07 \mathrm{E}-10$ & $\mathrm{~N} / \mathrm{A}$ & $4.35 \mathrm{E}-01$ & $2.50 \mathrm{E}-05$ & $5.59 \mathrm{E}-05$ \\
\hline Zoo & $3.80 \mathrm{E}-06$ & $1.55 \mathrm{E}-13$ & $\overline{2.71 \mathrm{E}-14}$ & $\mathrm{~N} / \mathrm{A}$ & $6.18 \mathrm{E}-04$ \\
\hline Clean1 & $1.09 \mathrm{E}-06$ & $4.81 \mathrm{E}-07$ & $\mathrm{~N} / \mathrm{A}$ & $2.15 \mathrm{E}-06$ & $1.94 \mathrm{E}-11$ \\
\hline semeion & $1.77 \mathrm{E}-11$ & $1.80 \mathrm{E}-11$ & $\mathrm{~N} / \mathrm{A}$ & $1.83 \mathrm{E}-11$ & $1.96 \mathrm{E}-11$ \\
\hline Colon & $9.45 \mathrm{E}-14$ & 2.39E-13 & $6.50 \mathrm{E}-14$ & $1.13 \mathrm{E}-13$ & $\mathrm{~N} / \mathrm{A}$ \\
\hline Leukemia & $3.09 \mathrm{E}-01$ & 4.63E-13 & $\mathrm{N} / \mathrm{A}$ & 3.31E-12 & $\mathrm{N} / \mathrm{A}$ \\
\hline
\end{tabular}


Table 8: Overall ranking results

\begin{tabular}{|c|c|c|c|c|c|c|c|c|c|c|c|c|c|c|}
\hline \multirow{2}{*}{$\begin{array}{l}\text { Algorithm } \\
\text { Metric }\end{array}$} & \multicolumn{3}{|c|}{ BGOA_S } & \multicolumn{3}{|c|}{ BGOA_EPD } & \multicolumn{3}{|c|}{ BGOA_EPD_CM } & \multicolumn{5}{|c|}{ BGOA_EPD_RWS BGOA_EPD_Tour } \\
\hline & Acc & Att & Fit & $\overline{A c c}$ & Att & Fit & $\overline{A c c}$ & Att & Fit & Acc & Fit & $\overline{A c c}$ & Att & Fit \\
\hline Breastcancer & 3 & 1 & 3 & 5 & 5 & 5 & 3 & 2 & 3 & 2 & & 1 & 4 & 1 \\
\hline BreastEW & 4 & 1 & 4 & 3 & 5 & 3 & 2 & 4 & 2 & & 1 & 5 & 3 & 5 \\
\hline Exactly & 5 & 5 & 5 & 3 & 4 & 3 & 4 & 3 & 4 & & 1 & 1 & 2 & 1 \\
\hline Exactly2 & 3 & 1 & 3 & 5 & 5 & 5 & 4 & 4 & 4 & 4 & 2 & 1 & 3 & 1 \\
\hline HeartEW & 4 & 4 & 4 & 1 & 3 & 1 & 2 & 1 & 2 & 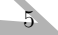 & 5 & 3 & 5 & 3 \\
\hline Lymphography & 5 & 1 & 5 & 1 & 2 & 1 & 4 & 2 & 4 & 2 & 2 & 3 & 4 & 3 \\
\hline M-of-n & 5 & 5 & 5 & 4 & 2 & 4 & 2 & 3 & 2 & 2 & 2 & 1 & 1 & 1 \\
\hline PenglungEW & 3 & 1 & 3 & 2 & 2 & 2 & 4 & 5 & 4 & 5 & 5 & 1 & 4 & 1 \\
\hline SonarEW & 4 & 1 & 4 & 2 & 4 & 2 & 5 & 3 & 5 & 1 & 1 & 3 & 5 & 3 \\
\hline SpectEW & 3 & 1 & 2 & 4 & 5 & 4 & 1 & 4 & 1 & 2 & 2 & 5 & 2 & 5 \\
\hline CongressEW & 5 & 1 & 5 & 1 & 5 & 2 & 3 & 4 & 3 & 1 & 1 & 4 & 3 & 4 \\
\hline IonosphereEW & 5 & 1 & 5 & 3 & 5 & 3 & 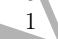 & 4 & 1 & 2 & 2 & 4 & 2 & 4 \\
\hline KrvskpEW & 5 & 1 & 5 & 3 & 4 & & & 3 & 4 & 2 & 2 & 1 & 2 & 1 \\
\hline Tic-tac-toe & 2 & 5 & 2 & 3 & 2 & 3 & 1 & 3 & 4 & 5 & 5 & 1 & 1 & 1 \\
\hline Vote & 4 & 1 & 4 & & & 5 & 3 & 4 & 3 & 2 & 2 & 1 & 2 & 1 \\
\hline WaveformEW & 5 & 1 & 5 & & 3 & 3 & 1 & 4 & 1 & 2 & 2 & 4 & 5 & 4 \\
\hline WineEW & 5 & 1 & & & 4 & 1 & 2 & 3 & 2 & 4 & 3 & 3 & 5 & 4 \\
\hline Zoo & 3 & 4 & & & & 4 & 5 & 2 & 5 & 1 & 1 & 2 & 5 & 2 \\
\hline Clean1 & 4 & 1 & & & $F$ & 2 & 1 & 5 & 1 & 2 & 2 & 5 & 2 & 5 \\
\hline Semeion & 4 & & & & 4 & 2 & 1 & 5 & 1 & 5 & 5 & 3 & 2 & 3 \\
\hline Colon & 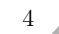 & & 4 & & 4 & 2 & 5 & 5 & 5 & 3 & 3 & 1 & 3 & 1 \\
\hline Leukemia & & & 3 & 4 & 4 & 4 & 1 & 5 & 2 & 5 & 5 & 1 & 2 & 1 \\
\hline Sum of the rank & & 40 & 86 & 63 & 84 & 64 & 62 & 78 & 63 & 57 & 59 & 54 & 67 & 55 \\
\hline Overall rank & & 1 & 5 & 3 & 5 & 4 & 3 & 4 & 3 & 2 & 2 & 1 & 3 & 1 \\
\hline Total sum & & & & 211 & & & 203 & & & 172 & & 176 & & \\
\hline Final ranl & & & & 4 & & & 3 & & & 1 & & 2 & & \\
\hline
\end{tabular}


and BGOA_EPD_Tour) have demonstrated very competitive performances and the ranks are 172 and 176, respectively. There is a notable gap between the ranks of top winners having the RWS and TS selection schemes and the third front-runner, the BGOA_EPD_CM that runs the mutation and crossover to relocate the feeble grasshoppers based on top front-runners of the population.

The reason might be that the BGOA_EPD_CM and BGOA_EPD have utilized the best grasshoppers of the population, and this can bias the exploration phase, which has instigated premature convergence and a loss of diversity in the population. This effect of best/solutions has decreased the quality of the outcomes, and consequently, the grades of the BGOA_EPD_CM and BGOA_EPD in the ranking system have dropped compared to the BGOA_EPD_Tour and BGOA_EPD RWS. From the other side, BGOA_EPD_Tour inherits the advantage of TS, which can preserve the diversity of grasshoppers and consequently, the BGOA_EPD_Tour has an advanced potential to retain and recover a stable balance between the exploration and exploitation inclinations. In addition, BGOA_EPD_Tour applies the mutation scheme to the selected grasshopper hoping to find a better solution in the neighbor of the selected solution and to avoid the LO. For these reasons, the BGOA_EPD_Tour can outperform all based on Acc and fitness measures.

Based on final ranks, the best version is the BGOA_EPD_RWS. The reason is that this version does not ignore any grasshopper in the population, so it is capable of preserving the diversity of the agents, which can help the BGOA_EPD_RWS to perform deeper exploration levels. This fact has enabled the BGOA_EPD_RWS to avoid LO and discover better results. In addition, after selecting an agent with the RWS operator, it is mutated to explore more areas of the feature space, then; the resulted solution is used again to reposition a solution from the second half by applying the crossover operator. these operators also improve the efficacy of the BGOA_EPD_RWS in balancing the exploration and exploitation as compared to other developed versions.

The convergence trends for the all proposed variants according to the fitness measure on all 22 datasets are also compared and demonstrated in Figs. 9 and 10.

From Fig. 9, it can be observed that the BGOA_EPD_RWS has exposed the best curves compared to other versions in tackling the BreastEW, CongressEW, Lymphography, and SonarEW problems. The BGOA_EPD_Tour 


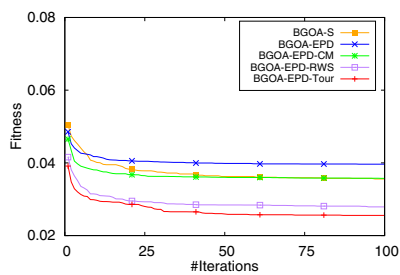

(a) Breastcancer

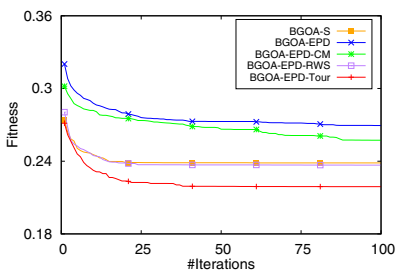

(d) Exactly2

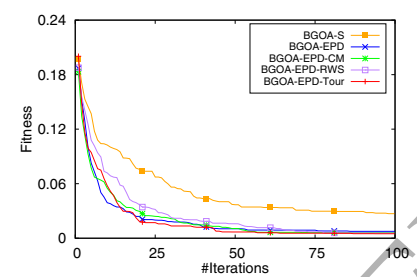

(g) M-of-n

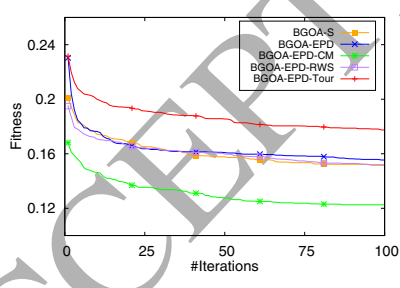

(j) SpectEW

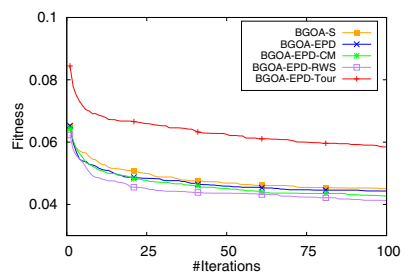

(b) BreastEW

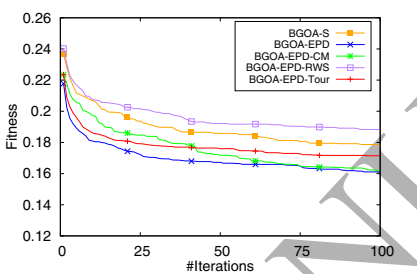

(e) HeartEW

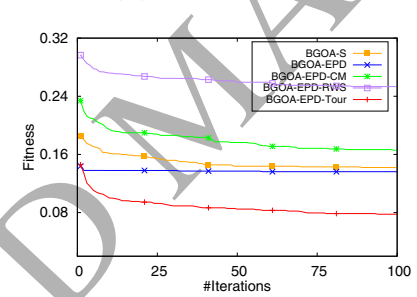

(h) penglungEW

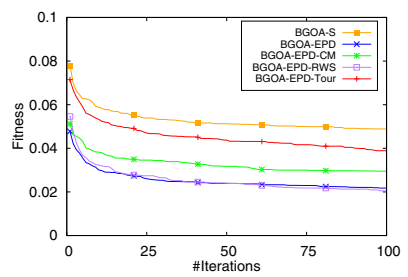

(k) CongressEW

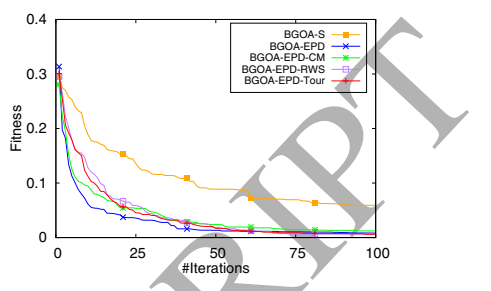

(c) Exactly

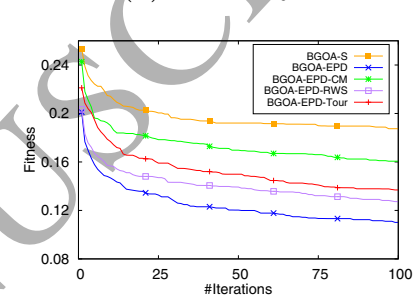

(f) Lymphography

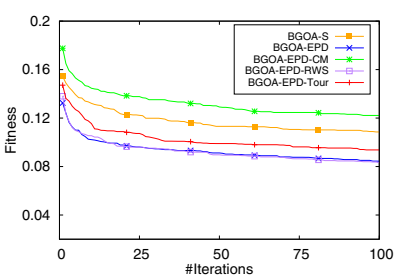

(i) SonarEW

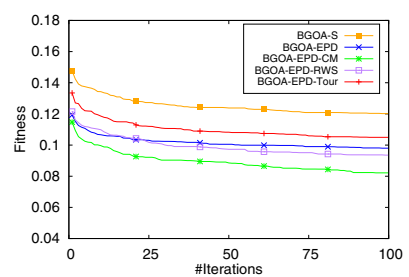

(1) IonosphereEW

Figure 9: Convergence curves of the proposed approaches for Breastcancer, BreastEW, Exactly, Exactly2, HeartEW, Lymphography, M-of-n, penglungEW, and SonarEW, SpectEW, CongressEW, and IonosphereEW datasets. 


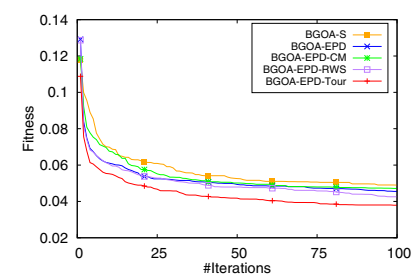

(a) KrvskpEW

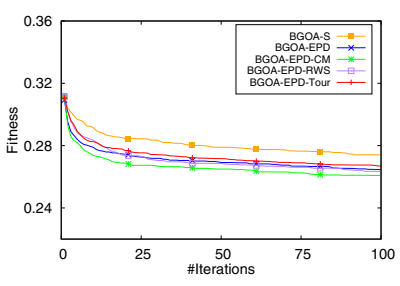

(d) WaveformEW

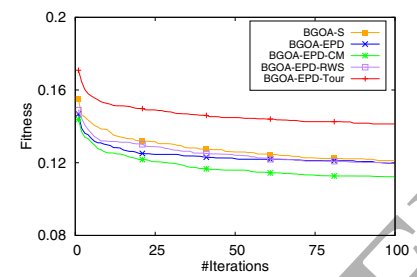

(g) Clean1

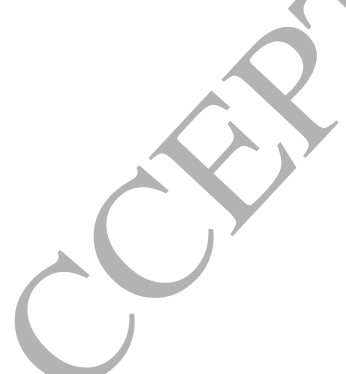

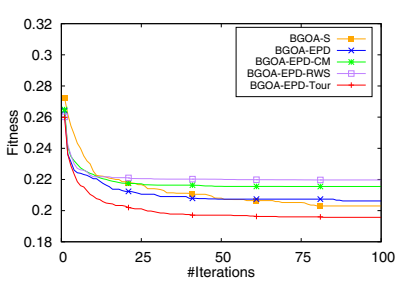

(b) Tic-tac-toe

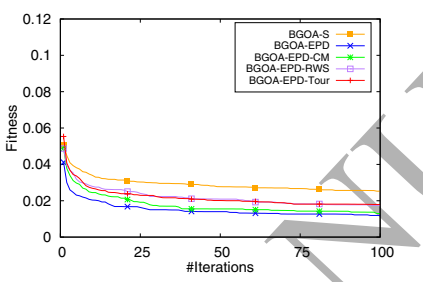

(e) WineEW

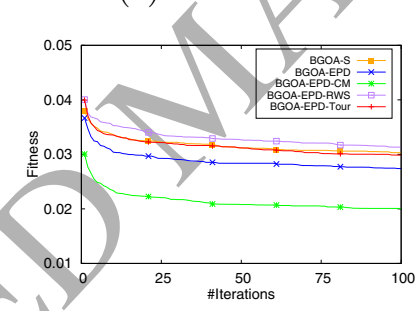

(h) Semeion

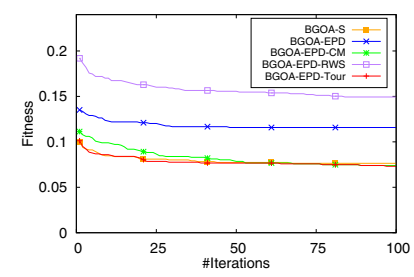

(j) Leukemia

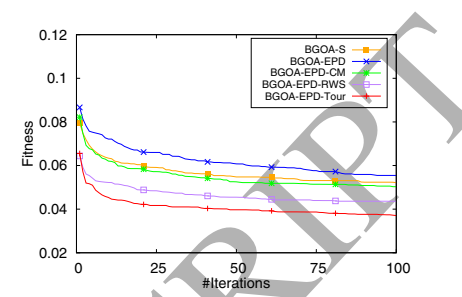

(c) Voté

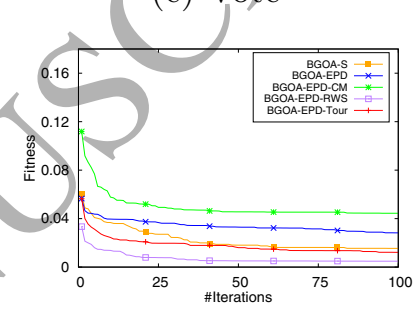

(f) Zoo

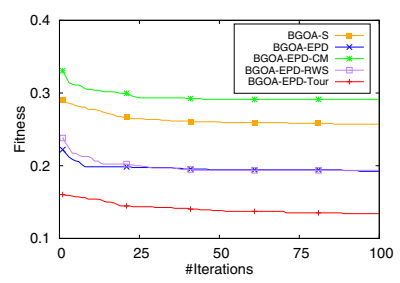

(i) Colon

Figure 10: Convergence curves of the proposed approaches for KrvskpEW, Tic-tac-toe, Vote, WaveformEW, WineEW, Zoo, Clean1, Semeion, Colon, and Leukemia datasets. 
can reveal a quicker tendency than others in treating the Breastcancer, Exactly2, HeartEW, KrvskpEW, M-of-n, and penglungEW datasets, while the BGOA_EPD has stagnated to LO in early steps of the exploration phase, for example when solving the Breastcancer, CongressEW, Exactly, HeartEW, IonosphereEW, KrvskpEW, Lymphography, and penglungEW tasks. From the curves on Fig. 9, it can be inferred that the approaches utilizing the TS and RWS schemes have a head-to-head convergence proclivity.

It seems that the TS and RWS-embedded variants perform better than versions employing random selection and top solutions for rearranging of the worst grasshoppers. This reveals that enhancing the median of all population using only top solutions and a random grasshopper may/increase the chance of BGOA_EPD to be easily captivated in LO when penetrating the fruitless regions of the feature space. The trends of the BGOA_EPD_CM also support that the crossover and mutation schemes has heightened the inclusive leaning of the BGOA_EPD in balancing the exploration and exploitation traits. Therefore, it is seen that the BGOA_EPD_CM can converge faster to better results than the $B_{G O A} \angle \mathrm{EPD}$ in basically all cases. And yet, the BGOA_EPD_CM cannot surpass both BGOA_EPD_Tour and BGOA_EPD_RWS in terms of conyergence results, except in solving the IonosphereEW. Therefore, it is seen that using selection mechanisms has alleviated the unripe convergence shortcoming of the BGOA_EPD. In RWS-based version, the door is open for the weak solution in the first half of the population to be selected but the better solutions have more chance. In this regard, the BGOA_EPD_Tour attains better results than BGOA_EPD_RWS.

According to Fig. 10, the convergence shortcomings of the BGOA_EPD can still be detected. However, it converged to improved results for the Clean1. The BGOA_S can be better than the BGOA_EPD regarding the convergence and it shows competitive trends for all cases.

The BGOA_EPD_CM has revealed enriched tendencies compared to the BGOA_EPD and BGOA_S, mainly on SpectEW, WaveformEW, WineEW, and Semeion problems, which has slightly outperformed other methods. It shows that the extra operators assist the BGOA_EPD in fleeing from the LO. When the TS-based selection theme has met the BGOA_EPD, it has shown best curves on the Tic-tac-toe, Vote, Colon, and Leukemia, whereas the BGOA_EPD_RWS has not exposed the excellent curves, except on the Zoo. The reason is that the TS can enhance and preserve the diversity of the solutions, which can encourage more stable balance between the local 
and global search trends. Then, it helps the BGOA_EPD_Tour to uncover superior trends on harder cases like the Leukemia with 7129 and Colon with 2000 features. It is seen that the tendencies of BGOA_EPD_Tour and BGOA_EPD_RWS are very competitive on the WineEW and WaveformEW.

Considering all results, convergence curves and final rankings of algorithms, it can be recognized that two best versions are the BGOA_EPD_RWS and BGOA_EPD_Tour algorithms. In the next section, the BGOA_EPD_Tour, which is the best hybrid variant of BGOA_EPD ,is considered to be further compared to other well-established optimizers with regard to the efficacy, performance on different metrics and convergence behaviors.

\subsection{Comparison with other metaheuristics}

In this section, the efficacy and qualitative results of the BGOA_EPD_Tour techniques is compared to the several well-regarded and related optimizers in the FS field from different aspects. The binary versions of the GWO (bGWO) [67], GSA (BGSA) [87], and BA (BBA) [88] are utilized here to deeply investigate the comparative efficiency of these well-established methods against the developed BGOA-based version.

All trials have completed during a same condition and all conditions were similar to the described info in preceding section. The parameters of optimizers are sensibly selected using many trial and error processes to comprehend the finest feasible settings. Table 9 shows the the used parameters.

Table 9: The parameter settings

\begin{tabular}{llll}
\hline & \multicolumn{3}{c}{ Table 9: The parameter settings } \\
\hline Algorithm & Parameter & Value \\
\hline GSA & $G_{0}$ & 100 \\
& $\alpha$ & 20 \\
& BA & $Q_{\min }$ Frequency minimum & 0 \\
& $Q_{\max }$ Frequency maximum & 2 \\
& $A$ Loudness & 0.5 \\
& & $r$ Pulse rate & 0.5 \\
& & $a$ & {$[20]$} \\
\hline
\end{tabular}

Table 10 exposes the attained Acc and related StdDev results for the proposed algorithms versus other metaheuristics. Tables 11-13 also reflect the average selected attributes, fitness, and CPU time values along with the related StdDev for the compared techniques. Table 14 shows the results of 
Wilcoxon sum rank statistical test for the accuracy results in Table 10. For the used test, the best technique for each dataset is considered as the base method to be compared with other peers, independently.

Table 10: Classification accuracy results of the BGOA_EPD_Tour compared to other metaheuristics

\begin{tabular}{|c|c|c|c|c|c|c|c|c|}
\hline \multirow{2}{*}{ Dataset } & \multicolumn{2}{|c|}{ BGOA_EPD_Tour } & \multicolumn{2}{|c|}{ bGWO } & \multicolumn{2}{|c|}{ BGSA } & \multicolumn{2}{|c|}{$\mathrm{BBA}$} \\
\hline & Acc & StdDev & Acc & StdDev & Acc & StdDev & Acc & StdDev \\
\hline Breastcancer & 0.980 & 0.001 & 0.968 & 0.002 & 0.957 & 0.004 & 0.937 & 0.031 \\
\hline BreastEW & 0.947 & 0.005 & 0.954 & 0.007 & 0.942 & 0.006 & 0.931 & 0.014 \\
\hline Exactly & 0.999 & 0.005 & 0.809 & 0.076 & 0.697 & 0.060 & 0.610 & 0.065 \\
\hline Exactly2 & 0.780 & 0.000 & 0.743 & 0.017 & 0.706 & 0.023 & 0.628 & 0.057 \\
\hline HeartEW & 0.833 & 0.004 & 0.792 & 0.017 & 0.777 & 0.022 & 0.754 & 0.033 \\
\hline Lymphography & 0.868 & 0.011 & 0.813 & 0.028 & & 0.022 & 0.701 & 0.069 \\
\hline M-of-n & 1.000 & 0.000 & 0.894 & 0.041 & 5 & 0.063 & 0.722 & 0.080 \\
\hline penglungEW & 0.927 & 0.013 & 0.850 & 0.01 & & 0.000 & 0.795 & 0.029 \\
\hline SonarEW & 0.912 & 0.009 & 0.836 & 0.016 & 0.888 & 0.015 & 0.844 & 0.036 \\
\hline SpectEW & 0.826 & 0.010 & 0.810 & 0.014 & 0.783 & 0.024 & 0.800 & 0.027 \\
\hline CongressEW & 0.964 & 0.005 & 0.948 & 0.01 & 0.951 & 0.008 & 0.872 & 0.075 \\
\hline IonosphereEW & 0.899 & 0.007 & 0.885 & 0.009 & 0.881 & 0.010 & 0.877 & 0.019 \\
\hline KrvskpEW & 0.968 & 0.003 & 0.934 & 0.015 & 0.908 & 0.048 & 0.816 & 0.081 \\
\hline Tic-tac-toe & 0.808 & 0.000 & & 0.032 & 0.753 & 0.024 & 0.665 & 0.063 \\
\hline Vote & 0.966 & 0.003 & 0.944 & 0.010 & 0.931 & 0.011 & 0.851 & 0.096 \\
\hline WaveformEW & 0.737 & 0.003 & 0.723 & 0.007 & 0.695 & 0.014 & 0.669 & 0.033 \\
\hline WineEW & 0.989 & 0.000 & 0.960 & 0.012 & 0.951 & 0.015 & 0.919 & 0.052 \\
\hline Zoo & 0.993 & 0.009 & 0.975 & 0.009 & 0.939 & 0.008 & 0.874 & 0.095 \\
\hline clean1 & & & 0.908 & 0.006 & 0.898 & 0.011 & 0.826 & 0.021 \\
\hline semeion & 0.976 & 02 & 0.972 & 0.003 & 0.971 & 0.002 & 0.962 & 0.006 \\
\hline Colon & 0.870 & 0.006 & 0.661 & 0.022 & 0.766 & 0.015 & 0.682 & 0.038 \\
\hline Leukemia & 0.931 & 0.014 & 0.884 & 0.016 & 0.844 & 0.014 & 0.877 & 0.029 \\
\hline
\end{tabular}

From Table 10, it is seen that the hybrid BGOA_EPD_Tour can evidently outperform all contestants on 20 datasets. The bGWO also outperform others on 2 problems: BreastEW and clean1. In comparison with the BGSA, the BGOA_EPD_Tour can provide better rates on a11 problems. In dealing with $2 \overline{1}$ datasets, the classification accuracies of the BGOA_EPD_Tour have improved in the interval of $0.5 \%$ (Semeion) to $30 \%$ (Exactly) in comparison with the BGSA. It can also outperform the BBA on all 22 datasets.

The best and worst average Acc that the bGWO have reached is $97.45 \%$ and $66.13 \%$ on Zoo and Colon datasets, respectively. For M-of-n case, the BGOA_EPD_Tour have reached to $100 \%$ accuracy, while bGWO has 
the accuracy of $89.41 \%$, which shows the superior efficacy of the proposed EPD-based optimizer. Based on the overall ranks, the BGOA_EPD_Tour has achieved to the first place and bGWO, BGSA, and BBA are the next choices, respectively. several substantial improvements in the StdDev index can also be detected. The main reason for improved efficacy of the BGOA_EPD_Tour is that the poor solutions are repositioned around the better ones in the BGOA_EPD_Tour using the EPD, and during this process, the selection mechanisms has assisted the proposed approach to maintain the diversity of swarm, and then, recover a fine balance between the exploration and exploitation. Therefore, in the case of stagnation to LO, they can escape from them using the random nature behind/ the utilized operators.

From the results reflected in Table 11, it is evident that the BBA algorithm is better than other algorithms on 19 datasets. The BGOA_EPD_Tour and BGSA algorithms have attained the next ranks. For CongressE $\bar{E}$ and Vote, is observed that the BGOA_EPD_Tour can show the best results.

Table 11: Average number of selected attributes results of the BGOA_EPD_Tour and BGOA_EPD_RWS compared to other metaheuristics

\begin{tabular}{|c|c|c|c|c|c|c|c|c|}
\hline \multirow{2}{*}{ Dataset } & \multicolumn{2}{|c|}{ BGOA_EPD_Tour } & \multicolumn{2}{|c|}{ bGWO } & \multicolumn{2}{|c|}{ BGSA } & \multicolumn{2}{|c|}{ BBA } \\
\hline & Att & StdDev & Att & StdDev & Att & StdDev & Att & StdDev \\
\hline Breastcancer & 5.000 & 0.000 & 7.100 & 1.447 & 6.067 & 1.143 & 3.667 & 1.373 \\
\hline BreastEW & 17.333 & & 19.000 & 4.307 & 16.567 & 2.979 & 12.400 & 2.762 \\
\hline Exactly & 6.533 & r & 10.233 & 1.654 & 8.733 & 1.048 & 5.733 & 1.893 \\
\hline Exactly2 & 1.533 & 0.507 & 7.333 & 4.155 & 5.100 & 2.107 & 6.067 & 2.333 \\
\hline HeartEW & 8.400 & 1.037 & 8.167 & 2.001 & 6.833 & 1.315 & 5.900 & 1.647 \\
\hline Lymphograp & & 1.217 & 11.100 & 1.971 & 9.167 & 1.895 & 7.800 & 2.203 \\
\hline M-of-n & 6.467 & 0.507 & 9.633 & 0.964 & 8.467 & 1.432 & 6.167 & 2.086 \\
\hline penglung & 178.333 & 15.486 & 166.333 & 28.232 & 157.167 & 7.729 & 126.167 & 15.601 \\
\hline SonarE & 36.767 & 4.240 & 36.233 & 8.613 & 30.033 & 3.700 & 24.700 & 5.377 \\
\hline & 11.100 & 3.044 & 12.633 & 2.442 & 9.533 & 2.300 & 7.967 & 2.282 \\
\hline & 5.767 & 2.012 & 7.300 & 2.136 & 6.767 & 2.402 & 6.233 & 2.063 \\
\hline IonosphereEW & 16.400 & 3.701 & 19.233 & 5.015 & 15.400 & 2.513 & 13.400 & 2.594 \\
\hline KrvskpEW & 21.667 & 2.496 & 27.367 & 3.388 & 19.967 & 2.125 & 15.000 & 2.853 \\
\hline Tic-tac-toe & 5.000 & 0.000 & 6.700 & 1.343 & 5.867 & 1.137 & 4.700 & 1.489 \\
\hline Vote) & 5.433 & 1.223 & 7.400 & 2.222 & 8.167 & 1.821 & 6.133 & 2.177 \\
\hline WaveformEW & 26.233 & 3.451 & 31.967 & 4.612 & 19.900 & 2.917 & 16.667 & 3.304 \\
\hline WineEW & 8.800 & 1.472 & 8.600 & 1.754 & 7.367 & 1.098 & 6.067 & 1.741 \\
\hline Zoo & 9.167 & 1.967 & 10.367 & 2.484 & 8.167 & 1.177 & 6.567 & 2.501 \\
\hline clean1 & 92.600 & 7.802 & 121.267 & 20.691 & 83.700 & 5.421 & 64.767 & 10.016 \\
\hline semeion & 157.033 & 11.485 & 200.100 & 31.022 & 133.533 & 7.422 & 107.033 & 10.947 \\
\hline Colon & 1063.667 & 64.618 & 1042.100 & 126.721 & 995.833 & 20.021 & 827.500 & 55.371 \\
\hline Leukemia & 3768.800 & 224.842 & 3663.767 & 294.872 & 3555.133 & 39.713 & 2860.000 & 247.642 \\
\hline
\end{tabular}


Inspecting the results in Table 12, it is observed that the proposed BGOA_EPD_Tour is capable of outperforming all algorithms and revealing the best costs in realizing 20 datasets.

The BGOA_EPD_Tour shows superior costs compared to the BBA, BGSA, and bGWO algorithms on $90.9 \%, 95.45 \%$, and $90.9 \%$ of the datasets, respectively. The reason is that the TS-based selection operator assists the algorithm to maintain the diversity of grasshoppers. It also utilizes top solutions to guide the poor ones and this strategy improves the exploitative behavior of algorithm. Hence, the BGOA_EPD_Tour an enriched potential to retain and recover a stable balance between the exploration and exploitation phases in dealing with difficult feature spaces. The effect of selection operators are seen in the background of the improved results.

The proposed BGOA_EPD_Tour has also attained an acceptable StdDev values. Regarding the fitness, the bGWO show a good efficacy on 2 datasets. The penglungEW, Colon, Tic-tac-toe, krrskpEW cases can be considered as relatively large datasets and the fitness values of the developed EPD-based version is relatively less than other optimizers.

Based on Table 13, the BGSA is the fastest approach and the binary BBA is placed at the next rank.

Table 14 shows all datasets that the proposed method provides the best results (20 cases), the improvements are meaningful and accuracy of classification results has significantly increased compared to the other competitors.

The convergence curves for the compared algorithms on all datasets are demonstrated in Figs. 11 and 12. It can be seen that the BGOA_EPD_Tour has an accelerated behavior on all 22 problems. Based on the last found solutions, which can be seen from the ending points of the curves, it can outperform all techniques in tackling 20 problems except the BreastEW and Clean1 datasets, which no stagnation behavior occurs but the concluding marks are not better than the bGWO algorithm. Premature convergence behaviors can be detected in the curves of the bGWO, BBA and BGSA in dealing with several cases including the Exactly, Vote, Tic-tac-toe, SpectEW, and Zoo cases. For 20 datasets, the curves BGOA_EPD_Tour are superior to those of other competitors. Based on the aforementioned remarks, it can be recognized that the novel EPD-based operators have strengthened the overall tradeoff between the exploratory and exploitative steps. Consequently, it alleviates the immature convergence drawbacks of the BGOA in dealing with FS problems. 
Table 12: Average fitness results of the BGOA_EPD_Tour and BGOA_EPD_RWS compared to other metaheuristics

\begin{tabular}{|c|c|c|c|c|c|c|c|c|}
\hline \multirow{2}{*}{ Dataset } & \multicolumn{2}{|c|}{ BGOA_EPD_Tour } & \multicolumn{2}{|c|}{ bGWO } & \multicolumn{2}{|c|}{ BGSA } & \multicolumn{2}{|c|}{$\mathrm{BBA}$} \\
\hline & Fitness & StdDev & Fitness & StdDev & Fitness & StdDev & Fitness & StdDev \\
\hline Breastcancer & 0.026 & 0.001 & 0.039 & 0.003 & 0.049 & 0.003 & 0.044 & 0.005 \\
\hline BreastEW & 0.058 & 0.004 & 0.051 & 0.007 & 0.063 & 0.006 & 0.056 & 0.006 \\
\hline Exactly & 0.006 & 0.006 & 0.197 & 0.077 & 0.307 & 0.059 & 0.323 & 0.074 \\
\hline Exactly2 & 0.219 & 0.000 & 0.260 & 0.019 & 0.295 & 0.024 & 0.326 & 0.017 \\
\hline HeartEW & 0.171 & 0.004 & 0.213 & 0.017 & 0.226 & 0.021 & 0.208 & 0.015 \\
\hline Lymphography & 0.137 & 0.011 & 0.191 & 0.028 & 0.222 & 0.022 & 0.226 & 0.024 \\
\hline M-of-n & 0.005 & 0.000 & 0.112 & 0.041 & 0.170 & 0.063 & 0.171 & 0.056 \\
\hline penglungEW & 0.078 & 0.012 & 0.154 & 0,013 & 0.085 & 0.000 & 0.168 & 0.017 \\
\hline SonarEW & 0.094 & 0.008 & 0.169 & 0.016 & 0.116 & 0.015 & 0.110 & 0.021 \\
\hline SpectEW & 0.177 & 0.010 & 0.194 & 0.014 & 0.220 & 0.024 & 0.172 & 0.012 \\
\hline CongressEW & 0.039 & 0.005 & 0.056 & 0.011 & 0.053 & 0.008 & 0.064 & 0.015 \\
\hline IonosphereEW & 0.105 & 0.007 & 0.120 & 0.009 & 0.122 & 0.010 & 0.108 & 0.012 \\
\hline KrvskpEW & 0.038 & 0.003 & 0.073 & 0.015 & 0.097 & 0.047 & 0.117 & 0.047 \\
\hline Tic-tac-toe & 0.196 & & 0.251 & 0.032 & 0.251 & 0.024 & 0.257 & 0.024 \\
\hline Vote & 0.037 & 0.003 & 0.060 & 0.010 & 0.073 & 0.011 & 0.071 & 0.013 \\
\hline WaveformEW & 0.267 & 0.003 & 0.283 & 0.007 & 0.307 & 0.014 & 0.304 & 0.014 \\
\hline WineEW & 0.018 & 0.001 & 0.047 & 0.012 & 0.054 & 0.015 & 0.036 & 0.013 \\
\hline Zoo & 012 & 0.008 & 0.032 & 0.009 & 0.065 & 0.008 & 0.042 & 0.015 \\
\hline clean1 & & 0.004 & 0.099 & 0.006 & 0.106 & 0.010 & 0.156 & 0.013 \\
\hline & 0.030 & 0.001 & 0.036 & 0.003 & 0.034 & 0.002 & 0.033 & 0.003 \\
\hline & 0.134 & 0.006 & 0.341 & 0.022 & 0.237 & 0.014 & 0.279 & 0.035 \\
\hline Leukemia & 0.073 & 0.014 & 0.120 & 0.016 & 0.160 & 0.013 & 0.085 & 0.023 \\
\hline
\end{tabular}


Table 13: Average CPU time (seconds) results of the BGOA_EPD_Tour and BGOA_EPD_RWS compared to other metaheuristics

\begin{tabular}{|c|c|c|c|c|c|c|c|c|}
\hline \multirow{2}{*}{ Dataset } & \multicolumn{2}{|c|}{ BGOA_EPD_Tour } & \multicolumn{2}{|c|}{ bGWO } & \multicolumn{2}{|c|}{ BGSA } & \multicolumn{2}{|c|}{$\mathrm{BBA}$} \\
\hline & Time & StdDev & Time & StdDev & Time & StdDev & Time & StdDev \\
\hline Breastcancer & 4.146 & 0.191 & 3.879 & 0.236 & 3.461 & 0.194 & 3.456 & 0.184 \\
\hline BreastEW & 5.404 & 0.241 & 4.602 & 0.234 & 3.748 & 0.173 & 3.862 & 0.196 \\
\hline Exactly & 6.014 & 0.277 & 6.154 & 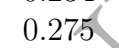 & 4.876 & 0.311 & 4.956 & 0.272 \\
\hline Exactly2 & 5.942 & 0.257 & 6.050 & 0.380 & 5.033 & 0.374 & 5.224 & 0.394 \\
\hline HeartEW & 3.505 & 0.178 & 2.739 & 0.174 & 2.812 & 0.182 & 2.767 & 0.201 \\
\hline Lymphography & 3.427 & 0.162 & 2.602 & 0.161 & 2.589 & 0.150 & 2.634 & 0.145 \\
\hline M-of-n & 6.173 & 0.271 & 6.157 & 0.241 & 5.138 & 0.293 & 4.892 & 0.361 \\
\hline penglungEW & 19.560 & 0.822 & 7.717 & 0.432 & 3.060 & 0.171 & 4.166 & 0.238 \\
\hline SonarEW & 5.801 & 0.245 & 3.677 & 0.193 & 2.703 & 0.146 & 2.850 & 0.191 \\
\hline SpectEW & 3.775 & 0.164 & 2.869 & 0.176 & 2.723 & 0.162 & 2.809 & 0.161 \\
\hline CongressEW & 4.109 & 0.205 & 3.309 & 0.172 & 3.217 & 0.188 & 3.244 & 0.158 \\
\hline IonosphereEW & 4.735 & 4.735 & 3.564 & 0.184 & 2.921 & 0.145 & 3.018 & 0.148 \\
\hline KrvskpEW & 57.043 & 1.743 & 78.113 & 4.606 & 49.534 & 2.527 & 47.923 & 2.576 \\
\hline Tic-tac-toe & 5007 & & 6.208 & 0.622 & 4.344 & 0.278 & 4.237 & 0.278 \\
\hline Vote & & 0.179 & 2.851 & 0.154 & 2.824 & 0.152 & 2.834 & 0.178 \\
\hline WaveformEW & 152.167 & 4.812 & 213.955 & 13.607 & 125.804 & 6.770 & 119.990 & 8.169 \\
\hline WineEW & & 0.301 & 2.628 & 0.173 & 2.585 & 0.171 & 2.624 & 0.141 \\
\hline Zoo & & 0.240 & 2.623 & 0.136 & 2.547 & 0.158 & 2.777 & 0.192 \\
\hline clean1 & & 0.608 & 13.683 & 0.647 & 7.397 & 0.288 & 7.589 & 0.467 \\
\hline & & 2.147 & 169.538 & 9.191 & 90.601 & 2.212 & 82.776 & 5.579 \\
\hline & 112.236 & 4.364 & 36.694 & 1.998 & 5.154 & 0.242 & 12.176 & 0.774 \\
\hline Leukemia & 394.688 & 15.418 & 130.878 & 6.366 & 16.441 & 0.683 & 39.379 & 2.418 \\
\hline
\end{tabular}


Table 14: P-values of the Wilcoxon test the classification accuracy results of BGOA_EPD_Tour and other meta heurstics algorithms $(\mathrm{p} \geq 0.05$ are underlined, and $\mathrm{N} / \mathrm{A}$ means not applicable).

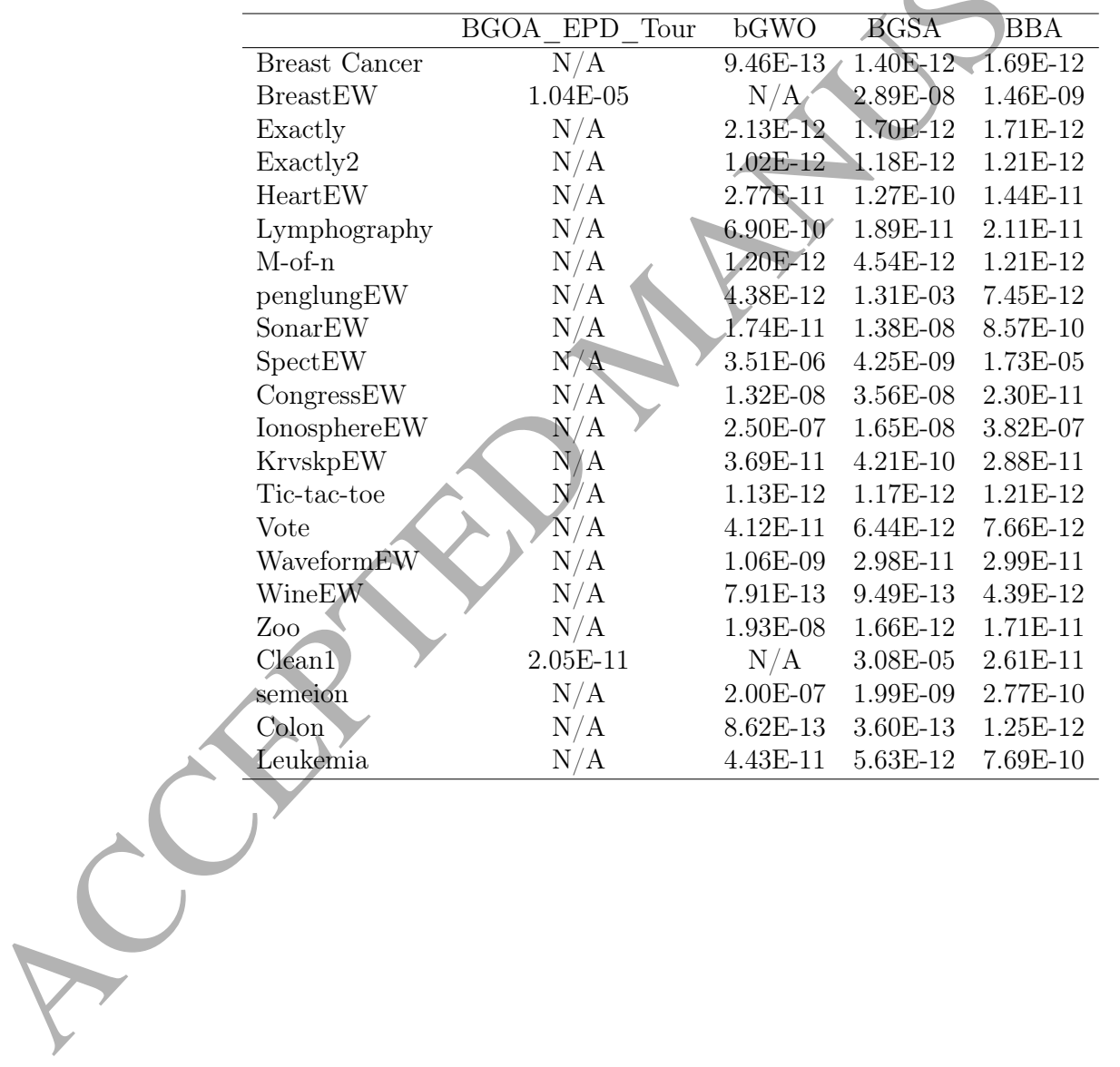




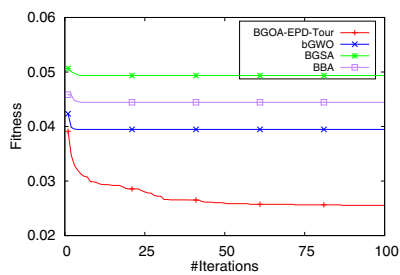

(a) Breastcancer

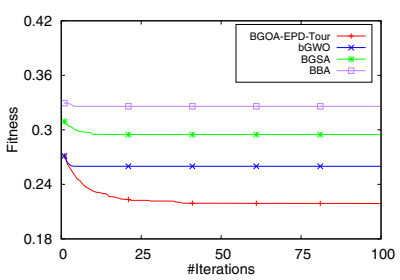

(d) Exactly2

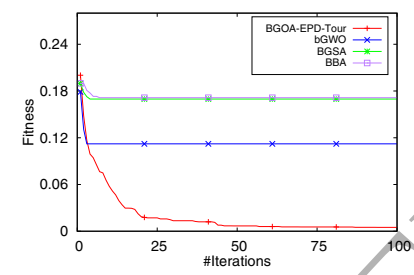

(g) M-of-n

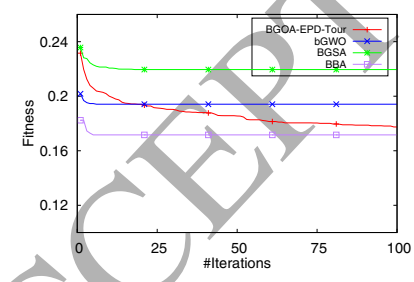

(j) SpectEW

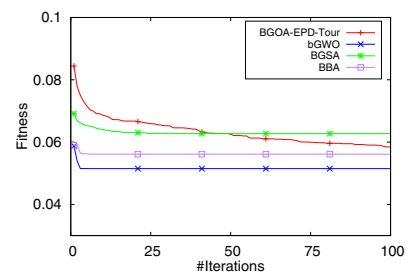

(b) BreastEW

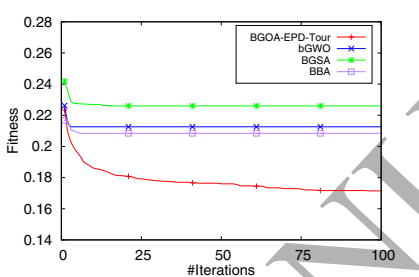

(e) HeartEW

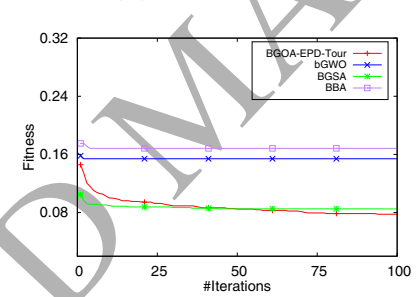

(h) penglungEW

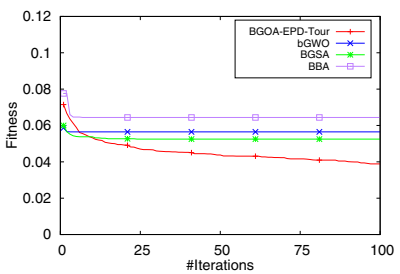

(k) CongressEW

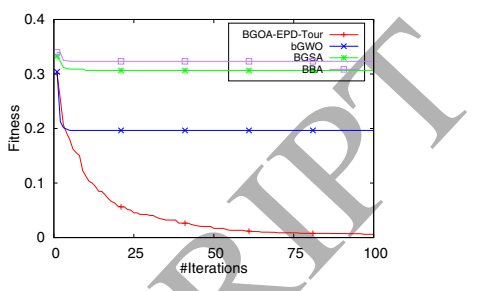

(c) Exactly

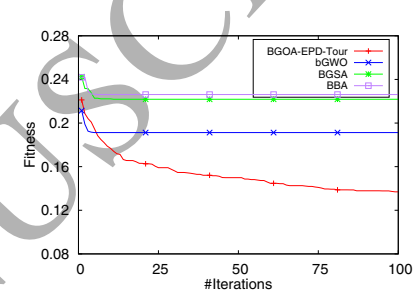

(f) Lymphography

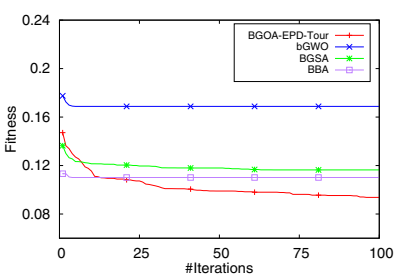

(i) SonarEW

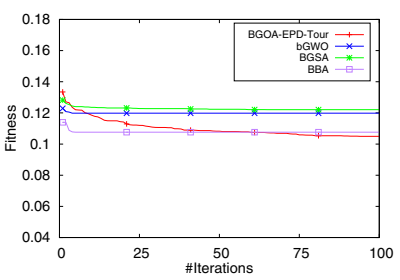

(l) IonosphereEW

Figure 11: Convergence curves for BGOA_EPD_Tour and other state-of-art methods for Breastcancer, BreastEW, Exactly, Exactly2, HeartEW, Lymphography, M-of-n, penglungEW, and SonarEW, SpectEW, CongressEW, and IonosphereEW datasets.. 


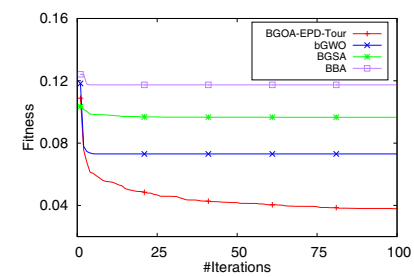

(a) KrvskpEW

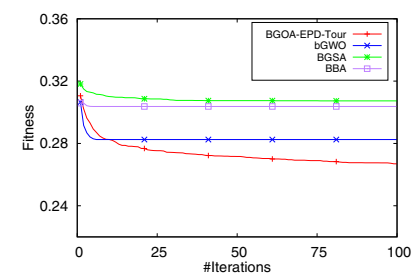

(d) WaveformEW

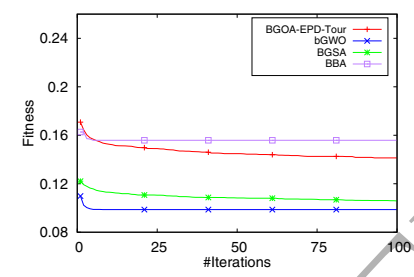

(g) Clean1

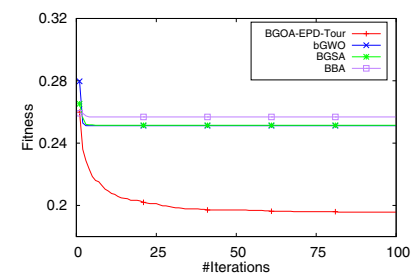

(b) Tic-tac-toe

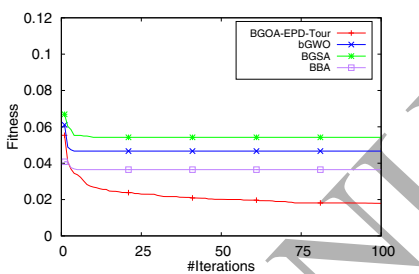

(e) WineEW

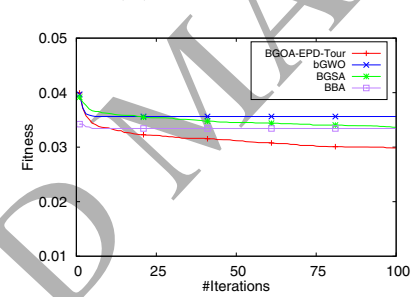

(h) Semeion

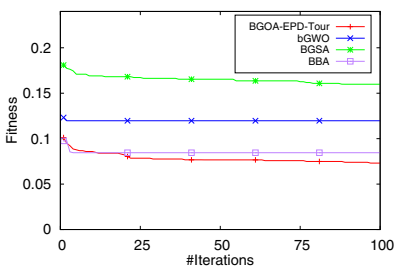

(j) Leukemia

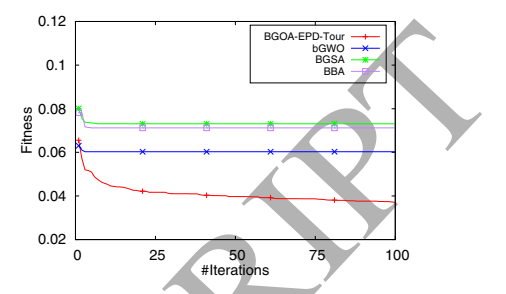

(c) Vote

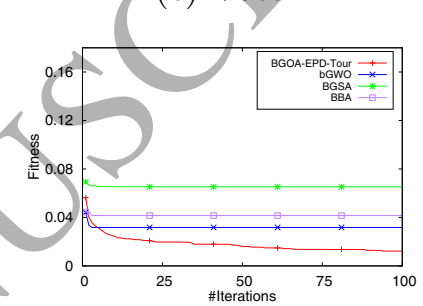

(f) Zoo

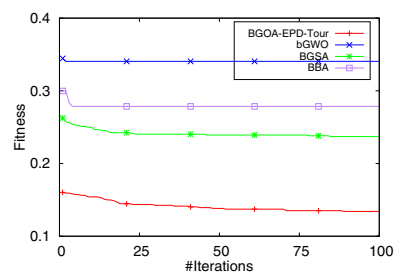

(i) Colon

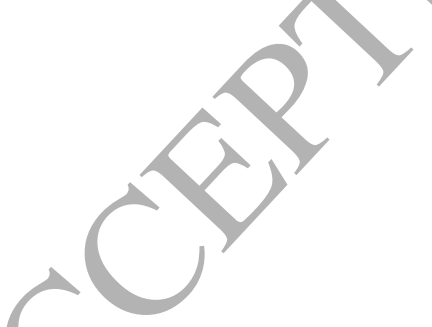

Figure 12: Convergence curves for BGOA EPD Tour and other state-of-art methods for KrvskpEW, Tic-tac-toe, Vote, WaveformEW, WineEW, Zoo, Clean1, Semeion, Colon, and Leukemia datasets. 


\subsection{Comparison with other meta-heuristics in the literature}

In this part, the classification rates of the proposed EPD-based approach are compared to some reported results from the past literature. Table 15 compares the average classification results of the BGOA_EPD_Tour with other algorithms obtained from the previous specialized works. The BGOA_EPD_Tour is compared to the reported classification results of GA and PSO from [89] and results of the bGWO1, bGWO2, GA, and PSO from [90]. Note that the first versions of GA and PSO were executed with exact settings in the implementation of the authors in [89]. While the results of other four approaches (bGWO1, bGWO2, GA, and PSO) in the table were obtained by the authors with the same datasets in [90].

Table 15: Classification accuracies of the BGOA_EPD_Tour versus other meta-heuristics from the specialized literature

\begin{tabular}{|c|c|c|c|c|c|c|c|}
\hline & BGOA_EPD_Tour & GA [89] & PSO [89] & bGWO1[90] & bGWO2 [90] & GA [90] & PSO [90] \\
\hline Breastcancer & 0.980 & 0.957 & 0.949 & 0.976 & 0.975 & 0.968 & 0.967 \\
\hline BreastEW & 0.947 & 0.923 & 0.933 & 0.924 & 0.935 & 0.939 & 0.933 \\
\hline Exactly & 0.999 & 0.822 & 0.973 & 0.708 & 0.776 & 0.674 & 0.688 \\
\hline Exactly2 & 0.780 & 0.677 & 0.666 & 0.745 & 0.750 & 0.746 & 0.730 \\
\hline HeartEW & 0.833 & 0.732 & 0.745 & 0.776 & 0.776 & 0.780 & 0.787 \\
\hline Lymphography & 0.868 & 0.758 & 0.759 & 0.744 & 0.700 & 0.696 & 0.744 \\
\hline M-of-n & 1.000 & 0.916 & 0.996 & 0.908 & 0.963 & 0.861 & 0.921 \\
\hline penglungEW & 0.927 & 0.672 & 0.879 & 0.600 & 0.584 & 0.584 & 0.584 \\
\hline SonarEW & 0.912 & 0.833 & 0.804 & 0.731 & 0.729 & 0.754 & 0.737 \\
\hline SpectEW & 0.826 & & 0.738 & 0.820 & 0.822 & 0.793 & 0.822 \\
\hline CongressEW & 0.964 & 0.898 & 0.937 & 0.935 & 0.938 & 0.932 & 0.928 \\
\hline IonosphereEW & 0.899 & 0.863 & 0.876 & 0.807 & 0.834 & 0.814 & 0.819 \\
\hline KrvskpEW & 0.968 & 0.940 & 0.949 & 0.944 & 0.956 & 0.920 & 0.941 \\
\hline Tic-tac-toe & 0.808 & 0.764 & 0.750 & 0.728 & 0.727 & 0.719 & 0.735 \\
\hline Vote & 0.966 & 0.808 & 0.888 & 0.912 & 0.920 & 0.904 & 0.904 \\
\hline WaveformEW & 0.737 & 0.712 & 0.732 & 0.786 & 0.789 & 0.773 & 0.762 \\
\hline WineEW & & 0.947 & 0.937 & 0.930 & 0.920 & 0.937 & 0.933 \\
\hline Zoo & 0.993 & 0.946 & 0.963 & 0.879 & 0.879 & 0.855 & 0.861 \\
\hline clean1 & & 0.862 & 0.845 & - & - & - & - \\
\hline semeion & & 0.963 & 0.967 & - & - & - & - \\
\hline Colon & 0.870 & 0.682 & 0.624 & - & - & - & - \\
\hline Leukemia & 0.931 & 0.705 & 0.862 & - & - & - & - \\
\hline
\end{tabular}

From the results in Table 15, it is evident that the attained classification rates by the developed EPD-based approach in this work are higher than other optimizers on 21 datasets and have a substantial superiority compared to those of GWO, GA, and PSO algorithms. The rates of the BGOA_EPD_Tour are better than the results of GA and PSO in [89] for all 22 datasets. In comparison with the revealed results in [90], the BGOA-based algorithm provides better classification rates than the PSO, GA, and bGWO1 
on all 18 available datasets and it can provide better than the bGWO2 on 17 datasets. These results also affirm that the proposed EPD-based and selection operators in the BGOA_EPD_Tour not only enriched its exploitation and exploitation capabilities and alleviated its stagnation problems but also enhanced the quality of the attained solutions for 22 datasets with various dimensions and characteristics.

\subsection{Comparison with filter-based techniques}

In this subsection, the classification of the EPD-embedded BGOA optimizer is compared to five well-known filter-based techniques [91]: correlationbased feature selection (CFS) [7], fast correlation-based filter (FCBF) [10], fisher score (F-score) [92], IG [93], and wavelet power spectrum (Spectrum) [11]. These filter-based techniques have carefully selected from two main classes: univariate and multivariate approaches. The IG, Spectrum, and F-Score are from the univariate strategies, which do not reflect the dependencies of the features in the assessment measure. In addition, CFS and FCBF are from the other category, which can employ the dependencies of the features. These approaches are investigated here because they have different mechanisms for utilizing the class labels of the training info to realize the relevance of analyzed features. The supervised approaches such as CFS, FCBF, F-Score and IG can utilize class labels whereas the unsupervised techniques such as Spectrum cannot handle labels for assessing the features. The results for the filter-based techniques after 20 runs are compared with the rates of the BGOA_EPD_Tour in Table 16. 
Table 16: Classification accuracy results of all filter-based methods versus the BGOA_EPD_Tour algorithm

\begin{tabular}{lcccccc}
\hline Dataset & CFS & FCBF & F-Score & IG & Spectrum & BGOA_EPD_Tour \\
\hline Breastcancer & 0.957 & $\mathbf{0 . 9 8 6}$ & 0.979 & 0.957 & 0.957 & 0.980 \\
BreastEW & 0.825 & 0.798 & 0.930 & 0.930 & 0.772 & $\mathbf{0 . 9 4 7}$ \\
Exactly & 0.670 & 0.440 & 0.600 & 0.615 & 0.575 & $\mathbf{0 . 9 9 9}$ \\
Exactly2 & 0.705 & 0.545 & 0.680 & 0.620 & 0.660 & $\mathbf{0 . 7 8 0}$ \\
HeartEW & 0.648 & 0.648 & 0.759 & 0.759 & 0.796 & $\mathbf{0 . 8 3 3}$ \\
Lymphography & 0.500 & 0.567 & 0.667 & 0.667 & 0.767 & $\mathbf{0 . 8 6 8}$ \\
M-of-n & 0.785 & 0.815 & 0.815 & 0.815 & 0.580 & $\mathbf{1 . 0 0 0}$ \\
PenglungEW & 0.600 & 0.667 & 0.800 & 0.667 & 0.400 & $\mathbf{0 . 9 2 7}$ \\
SonarEW & 0.310 & 0.214 & 0.048 & 0.191 & 0.048 & $\mathbf{0 . 9 1 2}$ \\
SpectEW & 0.736 & 0.774 & 0.793 & 0.793 & 0.736 & $\mathbf{0 . 8 2 6}$ \\
CongressEW & 0.793 & 0.793 & 0.908 & 0.828 & 0.828 & $\mathbf{0 . 9 6 4}$ \\
IonosphereEW & 0.857 & 0.857 & 0.729 & 0.800 & 0.829 & $\mathbf{0 . 8 9 9}$ \\
KrvskpEW & 0.768 & 0.934 & 0.959 & 0.934 & 0.377 & $\mathbf{0 . 9 6 8}$ \\
Tic-tac-toe & 0.000 & 0.000 & 0.010 & 0.010 & 0.167 & $\mathbf{0 . 8 0 8}$ \\
Vote & 0.950 & 0.950 & 0.933 & $\mathbf{0 . 9 6 7}$ & 0.850 & 0.966 \\
WaveformEW & 0.620 & 0.710 & 0.662 & 0.662 & 0.292 & $\mathbf{0 . 7 3 7}$ \\
WineEW & 0.778 & 0.889 & 0.861 & 0.889 & 0.889 & $\mathbf{0 . 9 8 9}$ \\
Zoo & 0.800 & 0.900 & 0.650 & 0.850 & 0.600 & $\mathbf{0 . 9 9 3}$ \\
clean1 & 0.716 & 0.642 & 0.632 & 0.547 & 0.611 & $\mathbf{0 . 8 6 3}$ \\
semeion & 0.875 & 0.875 & 0.875 & 0.868 & 0.875 & $\mathbf{0 . 9 7 6}$ \\
Colon & 0.750 & 0.667 & 0.667 & 0.667 & 0.500 & $\mathbf{0 . 8 7 0}$ \\
Leukemia & 0.929 & 0.857 & $\mathbf{0 . 9 8 0}$ & $\mathbf{0 . 9 8 0}$ & 0.357 & 0.931 \\
\hline
\end{tabular}

Inspecting the comparative results in Table 16, it is seen that the TSbased optimizer can outperform other algorithms on 17 datasets, while the IG and F-Score methods have obtained the best results for 2 datasets. It surpassed the supervised univariate approaches such as F-Score and IG, and the supervised multivariate types such as CFS and FCBF, and the unsupervised Spectrum technique. Furthermore, the results indicate that the wrapper-based FS procedures can provide superior rates in comparison with the filter-based versions since they can utilize both labels and dependencies during the selection of associated subsets. It can be concluded that the proposed algorithm has merits among other well-regarded optimizers and outperforms some well-known filter-based approaches.

Taken together, the results and discussions showed that the binary operators integrated into the BGOA algorithm were beneficial. The proposed operators slightly change the solutions that accelerates local search and convergence of the proposed algorithm. The EPD operator randomly changes the worst solutions, which promotes diversity and global search of the proposed algorithm. In feature selection problems, the shape of search space changes for every new dataset. Feature selection is normally considered for 
problems with medium or large number of features as well. To handle these difficulties, therefore, we need an efficient optimization algorithm that shows less local optima stagnation and high accuracy. Both operators proposed in the method assist BGOA_EPD in handling these difficulties.

\section{Conclusion and future directions}

In this study, an efficient GOA-based optimizer with EPD and selection operators was proposed to improve the efficacy of the basic GOA in dealing with FS tasks. The proposed GOA_EPD approaches were utilized extensively to tackle 22 benchmark datasets. The overall classification accuracy, selected features, fitness, consumed CPU time, and convergence behaviors of all hybrid versions were compared in detail to select the best version of the BGOA_EPD. The BGOA_EPD_Tour and BGOA_EPD_RWS has obtained the best place among four developed hybrid variants. The BGOA_EPD_Tour technique was utilized and compared in detail to various well-known metaheuristic-based and filter-based FS methods. The comprehensive comparative results and analysis revealed the improved efficacy of the proposed algorithm for solving different FS tasks.

Future studies can focus on the application of the EPD strategy to other population-based optimizers. The efficacy of the proposed binary GOA and EPD-based algorithms can also be employed to tackle other data mining problems. For future works, we intended to compare the proposed GOAEPD with different classes of FS methods in the field.

\section{References}

[1] Y. Huang, T. Li, C. Luo, H. Fujita, S.-j. Horng, Dynamic variable precision rough set approach for probabilistic set-valued information systems, Knowledge-Based Systems 122 (2017) 131-147.

[2] L. Jiao, F. Shang, F. Wang, Y. Liu, Fast semi-supervised clustering with enhanced spectral embedding, Pattern Recognition 45 (2012) 43584369.

[3] J. Han, J. Pei, M. Kamber, Data mining: concepts and techniques, Elsevier, 2011. 
[4] H. Wang, Z. Xu, W. Pedrycz, An overview on the roles of fuzzy set techniques in big data processing: Trends, challenges and opportunities, Knowledge-Based Systems 118 (2017) 15-30.

[5] H. Liu, H. Motoda, Feature Selection for Knowledge Discovery and Data Mining, Kluwer Academic Publishers, Boston, 1998.

[6] Y. Zhang, S. Wang, P. Phillips, G. Ji, Binary pso with mutation operator for feature selection using decision tree applied to spam detection, Knowledge-Based Systems 64 (2014) 22-31.

[7] M. A. Hall, L. A. Smith, Feature selection for machine learning: Comparing a correlation-based filter approach to the wrapper., in: FLAIRS conference, volume 1999, pp. 235-239.

[8] R. Shang, W. Wang, R. Stolkin, L. Jiao, Non-negative spectral learning and sparse regression-based dual-graph regularized feature selection, IEEE Transactions on Cybernetics (2017).

[9] F. Bellal, H. Elghazel, A. Aussem, A semi-supervised feature ranking method with ensemble learning, Pattern Recognition Letters 33 (2012) 1426-1433.

[10] L. Yu, H. Liu, Feature selection for high-dimensional data: A fast correlation-based filter solution, in: Proceedings of the 20th international conference on machine learning (ICML-03), pp. 856-863.

[11] Z. Zhao, H. Liu, Spectral feature selection for supervised and unsupervised learning, in: Proceedings of the 24th international conference on Machine learning, ACM, pp. 1151-1157.

[12] R. Shang, W. Wang, R. Stolkin, L. Jiao, Subspace learning-based graph regularized feature selection, Knowledge-Based Systems 112 (2016) 152165.

[13] R. Shang, Z. Zhang, L. Jiao, C. Liu, Y. Li, Self-representation based dual-graph regularized feature selection clustering, Neurocomputing 171 (2016) 1242-1253.

[14] J. Ren, Z. Qiu, W. Fan, H. Cheng, S. Y. Philip, Forward semi-supervised feature selection, in: Pacific-Asia conference on knowledge discovery and data mining, Springer, pp. 970-976. 
[15] M. Dash, H. Liu, Feature selection for classification, Intelligent data analysis 1 (1997) 131-156.

[16] V. Bolón-Canedo, N. Sánchez-Maroño, A. Alonso-Betanzos, Recent advances and emerging challenges of feature selection in the context of big data, Knowledge-Based Systems 86 (2015) 33-45.

[17] H. Liu, R. Setiono, Chi2: Feature selection and discretization of numeric attributes, 1995.

[18] J. Quinlan, Induction of decision trees, Machine learning 1 (1986) 81106.

[19] J. Quinlan, C4. 5: programs for machine learning, Morgan kaufmann, 1993.

[20] M. Robnik-Øě ikonja, I. Kononenko, Theoretical and empirical analysis of relieff and rrelieff, Machine learning 53 (2003) 23-69.

[21] A. Wang, N. An, G. Chen, L. Li, G.Alterovitz, Accelerating wrapperbased feature selection with k-nearest-neighbor, Knowledge-Based Systems 83 (2015) 81-91.

[22] H. Liu, R. Setiono, Eeature selection and classification-a probabilistic wrapper approach, 1996.

[23] R. Setiono, H. Liu, Neural-network feature selector, Neural Networks, IEEE Transactions on 8 (1997) 654-662.

[24] W. Siedlecki, J. Sklansky, On automatic feature selection, International Journal of Pattern Recognition and Artificial Intelligence 2 (1988) 197220.

[25] P. Langley, Selection of relevant features in machine learning, 1994.

[26] C. Lai, M. J. Reinders, L. Wessels, Random subspace method for multivariate feature selection, Pattern recognition letters 27 (2006) 10671076.

[27] E. Talbi, Metaheuristics From design to implementation, Wiley Online Library, 2009. 
[28] S. Saremi, S. Z. Mirjalili, S. M. Mirjalili, Evolutionary population dynamics and grey wolf optimizer, Neural Computing and Applications 26 (2015) 1257-1263.

[29] S. Mirjalili, S. M. Mirjalili, A. Lewis, Grey wolf optimizer, Advances in Engineering Software 69 (2014) 46-61.

[30] S. Mirjalili, A. Lewis, The whale optimization algorithm, Advances in Engineering Software 95 (2016) 51-67.

[31] S. Mirjalili, The ant lion optimizer, Advances in Engineering Software 83 (2015) 80-98.

[32] X.-S. Yang, Firefly Algorithms for Multimodal Optimization, Springer Berlin Heidelberg, Berlin, Heidelberg, pp. 169-178.

[33] J. Kennedy, R. Eberhart, A new optimizer using particle swarm theory, in: Micro Machine and Human Science, 1995. MHS '95., Proceedings of the Sixth International Symposium on, pp. 39-43.

[34] M. Dorigo, M. Birattari, T. Stutzle, Ant colony optimization, IEEE computational intelligence magazine 1 (2006) 28-39.

[35] I. Guyon, A. Elisseeff An introduction to variable and feature selection, Journal of machine learning research 3 (2003) 1157-1182.

[36] E. ZorarpacØdÂś, S. A. ØčâĂŞzel, A hybrid approach of differential evolution and artificial bee colony for feature selection, Expert Systems with Applications 62 (2016) 91-103.

[37] J. Wu, H. Wang, N. Li, P. Yao, Y. Huang, H. Yang, Path planning for solar-powered uav in urban environment, Neurocomputing (2017).

[38] W. Hang, K.-S. Choi, S. Wang, Synchronization clustering based on central force optimization and its extension for large-scale datasets, Knowledge-Based Systems 118 (2017) 31-44.

[39] Y. Z. Li, L. Jiang, Q. Wu, P. Wang, H. B. Gooi, K. Li, Y. Liu, P. Lu, M. Cao, J. Imura, Wind-thermal power system dispatch using mlsad model and gsoiclw algorithm, Knowledge-Based Systems 116 (2017) 94-101. 
[40] E. Ali, S. A. Elazim, A. Abdelaziz, Ant lion optimization algorithm for optimal location and sizing of renewable distributed generations, Renewable Energy 101 (2017) 1311-1324.

[41] H. Faris, I. Aljarah, M. A. Al-Betar, S. Mirjalili, Grey wolf optimizer: a review of recent variants and applications, Neural Computing and Applications (2017).

[42] K. Sorensen, M. Sevaux, F. Glover, A history of metaheuristics, arXiv preprint arXiv:1704.00853 (2017).

[43] S. Saremi, S. Mirjalili, A. Lewis, Grasshopper optimisation algorithm: Theory and application, Advances in Engineering Software 105 (2017) $30-47$.

[44] A. A. El-Fergany, Electrical characterisation of proton exchange membrane fuel cells stack using grasshopper optimiser, IET Renewable Power Generation (2017).

[45] J. Wu, H. Wang, N. Li, P. Yao, Y. Huang, Z. Su, Y. Yu, Distributed trajectory optimization for multiple solar-powered uavs target tracking in urban environment by adaptive grasshopper optimization algorithm, Aerospace Science and Technology 70 (2017) 497-510.

[46] A. Tharwat, E. H.Houssein, M. M. Ahmed, A. E. Hassanien, T. Gabel, Mogoa algorithm for constrained and unconstrained multi-objective optimization problems, Applied Intelligence (2017) 1-16.

[47] S. Z. Mirjalili, S. Mirjalili, S. Saremi, H. Faris, I. Aljarah, Grasshopper optimization algorithm for multi-objective optimization problems, Applied Intelligence (2017) 1-16.

[48] S. Mirjalili, Sca: a sine cosine algorithm for solving optimization problems, Knowledge-Based Systems 96 (2016) 120-133.

[49] T. BØčÂd’ck, D. Fogel, Z. Michalewicz, Handbook of evolutionary computation, Release 97 (1997) B1.

[50] A.-C. Zăvoianu, E. Lughofer, W. Koppelstätter, G. Weidenholzer, W. Amrhein, E. P. Klement, Performance comparison of generational and steady-state asynchronous multi-objective evolutionary algorithms 
for computationally-intensive problems, Knowledge-Based Systems 87 (2015) 47-60.

[51] J. H. Holland, Adaptation in natural and artificial systems, MIT Press, 1992.

[52] L. J. Fogel, A. J. Owens, M. J. Walsh, Artificial intelligence through simulated evolution, Wiley, 1966.

[53] C.-F. Tsai, W. Eberle, C.-Y. Chu, Genetic algorithms in feature and instance selection, Knowledge-Based Systems 39 (2013) 240-247.

[54] P. Moradi, M. Rostami, Integration of graph clustering with ant colony optimization for feature selection, Knowledge-Based Systems 84 (2015) 144-161.

[55] H. Wang, X. Jing, B. Niu, A discrete bacterial algorithm for feature selection in classification of microarray gene expression cancer data, Knowledge-Based Systems 126 (2017) 8-19.

[56] R. Jensen, Q. Shen, Finding rough set reducts with ant colony optimization, in: Proceedings of the 2003 UK workshop on computational intelligence, volume 1, pp. 15-22.

[57] R. Jensen, Q. Shen, Semantics-preserving dimensionality reduction: Rough and fuzzy-rough-based approaches, IEEE Trans. on Knowl. and Data Eng. 16 (2004) 1457-1471.

[58] H. Chen, W. Jiang, C. Li, R. Li, A heuristic feature selection approach for text categorization by using chaos optimization and genetic algorithm, Mathematical problems in Engineering 2013 (2013).

[59] M. Mafarja, S. Abdullah, Investigating memetic algorithm in solving rough set attribute reduction, International Journal of Computer Applications in Technology 48 (2013) 195-202.

[60] M. Majdi, S. Abdullah, N. S. Jaddi, Fuzzy population-based metaheuristic approaches for attribute reduction in rough set theory, World Academy of Science, Engineering and Technology, International Journal of Computer, Electrical, Automation, Control and Information Engineering 9 (2015) 2289-2297. 
[61] J. Wang, A.-R. Hedar, S. Wang, Scatter search for rough set attribute reduction, in: Bio-Inspired Computing: Theories and Applications, 2007. BIC-TA 2007. Second International Conference on, IEEE, pp. 236240.

[62] S. Mirjalili, The ant lion optimizer, Advances in Engineering Søftware 83 (2015) 80-98.

[63] E. Emary, H. M. Zawbaa, A. E. Hassanien, Binary antlion approaches for feature selection, Neurocomputing (2016).

[64] H. M. Zawbaa, E. Emary, B. Parv, Feature selection based on antlion optimization algorithm, 2015.

[65] H. M. Zawbaa, E. Emary, C. Grosan, Feature selection via chaotic antlion optimization, PloS one 11 (2016) e0150652.

[66] R.-E. Precup, R.-C. David, E. M. Petriu, Grey wolf optimizer algorithmbased tuning of fuzzy control systems with reduced parametric sensitivity, IEEE Transactions on Industrial Electronics 64 (2017) 527-534.

[67] E. Emary, H. M. Zawbaa, A. E. Hassanien, Binary grey wolf optimization approaches for featureselection, Neurocomputing 172 (2016) $371-381$.

[68] E. Emary, H. M. Zawbaa, C. Grosan, A. E. Hassenian, Feature subset selection approach by gray-wolf optimization, in: Afro-European Conference for Industrial Advancement, Springer, pp. 1-13.

[69] M. M.Mafarja, S. Mirjalili, Hybrid whale optimization algorithm with simulated annealing for feature selection, Neurocomputing (2017).

[70] A. Lewis, S. Mostaghim, M. Randall, Evolutionary population dynamics and multi-objective optimisation problems, Multi-Objective Optimization in Computational Intelligence: Theory and Practice (2008) $185-206$.

[71] S. Boettcher, A. G. Percus, Extremal optimization: Methods derived from co-evolution, in: Proceedings of the 1st Annual Conference on Genetic and Evolutionary Computation-Volume 1, Morgan Kaufmann Publishers Inc., 1999, pp. 825-832. 
[72] M. Randall, T. Hendtlass, A. Lewis, Extremal optimisation for assignment type problems, Biologically-Inspired Optimisation Methods: Parallel Algorithms, Systems and Applications 210 (2009) 139-164.

[73] D. Xie, Z. Luo, F. Yu, The computing of the optimal power consumption for semi-track air-cushion vehicle using hybrid generalized extremal optimization, Applied Mathematical Modelling 33 (2009) 2831-2844.

[74] K. Tamura, H. Kitakami, A. Nakada, Distributed modified extremal optimization using island model for reducing crossovers in reconciliation graph, Engineering Letters 21 (2013).

[75] P. Bak, C. Tang, K. Wiesenfeld, Self-organized criticality: An explanation of the $1 /$ f noise, Physical review letters 59 (1987) 381.

[76] A. Lewis, D. Abramson, T. Peachey, An evolutionary programming algorithm for automatic engineering design, in: International Conference on Parallel Processing and Applied Mathematics, Springer, pp. 586-594.

[77] A. A. Heidari, P. Pahlavani, An efficient modified grey wolf optimizer with lévy flight for optimization tasks, Applied Soft Computing 60 (2017) 115-134.

[78] A. A. Heidari, R. A. Abbaspour, A. R. Jordehi, An efficient chaotic water cycle algorithm for optimization tasks, Neural Computing and Applications 28 (2017) 57-85.

[79] S. K. Pal, A. Skowron, Rough-fuzzy hybridization: A new trend in decision making, Springer-Verlag New York, Inc., 1999.

[80] S. Mirjalili, A. Lewis, S-shaped versus v-shaped transfer functions for binary particle swarm optimization, Swarm and Evolutionary Computation 9 (2013) 1-14.

[81] J.) Kennedy, R. C. Eberhart, A discrete binary version of the particle swarm algorithm, in: Systems, Man, and Cybernetics, 1997. Computational Cybernetics and Simulation., 1997 IEEE International Conference on, volume 5, IEEE, pp. 4104-4108.

[82] N. S. Altman, An introduction to kernel and nearest-neighbor nonparametric regression, The American Statistician 46 (1992) 175-185. 
[83] T. Back, Evolutionary algorithms in theory and practice: evolution strategies, evolutionary programming, genetic algorithms, Oxford university press, 1996.

[84] B. L. Miller, D. E. Goldberg, et al., Genetic algorithms, tournament selection, and the effects of noise, Complex systems 9 (1995) 193-212.

[85] E. Emary, H. M. Zawbaa, A. E. Hassanien, Binary ant lion approaches for feature selection, Neurocomputing 213 (2016) 54-65.

[86] J. Friedman, T. Hastie, R. Tibshirani, The elements of statistical learning, volume 1, Springer series in statistics New York, 2001.

[87] E. Rashedi, H. Nezamabadi-Pour, S. Saryazdí, Bgsa: binary gravitational search algorithm, Natural Computing 9 (2010) 727-745.

[88] S. Mirjalili, S. M. Mirjalili, X.-S. Yang, Binary bat algorithm, Neural Computing and Applications 25 (2014) 663-681.

[89] S. Kashef, H. Nezamabadi-pour, An advanced aco algorithm for feature subset selection, Neurocomputing 147 (2015) 271-279.

[90] E. Emary, H. M. Zawbaa, A. E. Hassanien, Binary grey wolf optimization approaches for feature selection, Neurocomputing 172 (2016) $371-381$.

[91] Y. Saeys, I. Inza, P. Larrañaga, A review of feature selection techniques in bioinformatics, bioinformatics 23 (2007) 2507-2517.

[92] R. O. Duda, P. E. Hart, D. G. Stork, Pattern classification, John Wiley \& Sons, 2012.

[93] T. M. Cover, J. A. Thomas, Elements of information theory, John Wiley \& Sons, 2012. 


\section{Accepted Manuscript}

Evolutionary Population Dynamics and Grasshopper Optimization Approaches for Feature Selection Problems

Majdi Mafarja, Ibrahim Aljarah, Ali Asghar Heidari, Abdelaziz I. Hammouri, Hossam Faris, Ala'M. Al-Zoubi, Seyedali Mirjalili

PII:

DOI:

Reference:

To appear in:

Received date:

Revised date:

Accepted date:
S0950-7051(17)30615-9

10.1016/j.knosys.2017.12.037

KNOSYS 4173

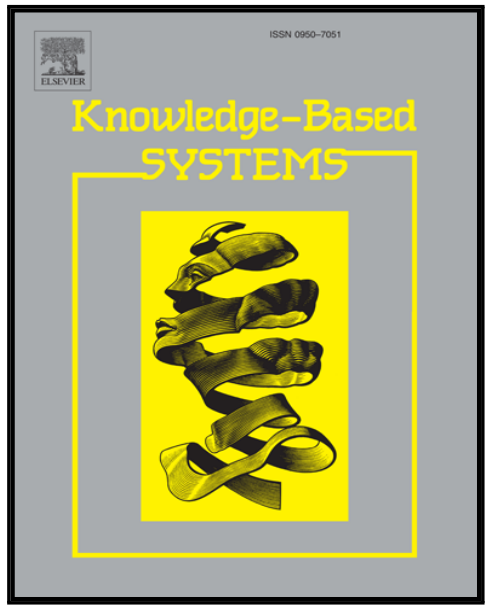

Please cite this article as: Majdi Mafarja, Ibrahim Aljarah, Ali Asghar Heidari, Abdelaziz I. Hammouri, Hossam Faris, Ala'M. Al-Zoubi, Seyedali Mirjalili, Evolutionary Population Dynamics and Grasshopper Optimization Approaches for Feature Selection Problems, Knowledge-Based Systems (2017), doi: 10.1016/j.knosys.2017.12.037

This is a PDF file of an unedited manuscript that has been accepted for publication. As a service to our customers we are providing this early version of the manuscript. The manuscript will undergo copyediting, typesetting, and review of the resulting proof before it is published in its final form. Please note that during the production process errors may be discovered which could affect the content, and all legal disclaimers that apply to the journal pertain. 